\title{
Layer- and Cell Type-Specific Response Properties of Gustatory Cortex Neurons in Awake Mice
}

\author{
Gulce Nazli Dikecligil, Dustin M. Graham, ${ }^{-}$Il Memming Park, and Alfredo Fontanini \\ Department of Neurobiology and Behavior and Program in Neuroscience, State University of New York at Stony Brook, Stony Brook, \\ New York 11794
}

Studies in visual, auditory, and somatosensory cortices have revealed that different cell types as well as neurons located in different laminae display distinct stimulus response profiles. The extent to which these layer and cell type-specific distinctions generalize to gustatory cortex (GC) remains unknown. In this study, we performed extracellular recordings in adult female mice to monitor the activity of putative pyramidal and inhibitory neurons located in deep and superficial layers of GC. Awake, head-restrained mice were trained to lick different tastants (sucrose, salt, citric acid, quinine, and water) from a lick spout. We found that deep layer neurons show higher baseline firing rates (FRs) in GC with deep-layer inhibitory neurons displaying highest FRs at baseline and following the stimulus. GC's activity shows robust modulations before animals' contact with tastants, and this phenomenon is most prevalent in deep-layer inhibitory neurons. Furthermore, we show that licking activity strongly shapes the spiking pattern of GC pyramidal neurons, eliciting phase-locked spiking across trials and tastants. We demonstrate that there is a greater percentage of taste-coding neurons in deep versus superficial layers with chemosensitive neurons across all categories showing similar breadth of tuning, but different decoding performance. Lastly, we provide evidence for functional convergence in GC, with neurons that can show prestimulus activity, licking-related rhythmicity and taste responses. Overall, our results demonstrate that baseline and stimulus-evoked firing profiles of GC neurons and their processing schemes change as a function of cortical layer and cell type in awake mice.

Key words: behavior; cortex; insular cortex; licking; taste

Significance Statement

Sensory cortical areas show a laminar structure, with each layer composed of distinct cell types embedded in different circuits. While studies in other primary sensory areas have elucidated that pyramidal and inhibitory neurons belonging to distinct layers show distinct response properties, whether and how response properties of gustatory cortex (GC) neurons change as a function of their laminar position and cell type remains uninvestigated. Here, we show that there are several notable differences in baseline, prestimulus, and stimulus-evoked response profiles of pyramidal and inhibitory neurons belonging to deep and superficial layers of GC.

\section{Introduction}

A great deal of research has focused on understanding how circuits in different layers of sensory cortices process sensory information. Studies in the somatosensory, auditory, and visual systems provide clear evidence of a laminar-specificity of sensory

\footnotetext{
Received July 3, 2019; revised Feb. 27, 2020; accepted 0ct. 24, 2020.

Author contributions: G.N.D., D.M.G., and A.F. designed research; G.N.D. and D.M.G. performed research; I.M.P. contributed unpublished reagents/analytic tools; G.N.D. analyzed data; G.N.D. and A.F. wrote the manuscript.

This work was supported by the National institute of Deafness and Other Communication Disorders Grant R01DC015234 (to A.F.) and the National Research Service Award Grant 1F32DC012461-01A1 (to D.M.G.). We thank Dr. Luca Mazzucato, Dr. Roberto Vincis, Dr. Haixin Liu, and Dr. Ke Chen for their helpful comments and discussions.

The authors declare no competing financial interests.

Correspondence should be addressed to Gulce Nazli Dikecligil at nazdikec@pennmedicine.upenn.edu.

https://doi.org/10.1523/JNEUROSCI.1579-19.2020

Copyright $(2020$ the authors
}

coding (Gilbert, 1977; Martinez et al., 2002; Krupa et al., 2004; de Kock et al., 2007; Poo and Isaacson, 2009; Sakata and Harris, 2009; Crochet et al., 2011; Reyes-Puerta et al., 2015). For instance, responses in layer 4 (L4) and L2/3 tend to be shorter and weaker than responses in the deep layers of rodent somatosensory cortex. In addition, only neurons in deep layers show characteristic anticipatory patterns of activity, which precede sensory stimulation (Krupa et al., 2004). In the auditory cortex, tones and clicks evoke sparse responses in superficial layers and dense responses in L5 neurons (Sakata and Harris, 2009). In the visual cortex, the distribution of complex and simple receptive fields changes in a layer-dependent manner (Martinez et al., 2002).

While the laminar distribution of sensory responses has been extensively studied in most sensory cortices, not much is known regarding the gustatory cortex (GC). Understanding how neurons in different layers are modulated during active tasting is 
particularly important given the heterogeneity of GC responses observed in alert rodents. Indeed, GC neurons can modulate their firing rates (FRs) in anticipation of taste (Stapleton et al., 2007; Samuelsen et al., 2012), fire rhythmically with licking (Stapleton et al., 2006; Gutierrez et al., 2010) and produce temporally dynamic responses encoding taste quality (Jones et al., 2007; Jezzini et al., 2013). Whether any of these patterns are uniformly distributed across the various layers or clustered in specific parts of the circuit is unknown. Furthermore, no information is available on how excitatory and inhibitory interneurons respond differently in the context of a tasting task. While we hypothesize to observe laminar and cellular differences in firing properties and processing schemes, we also predict, based on GC interconnectivity, to observe a significant degree of multiplexing.

Classic studies in anesthetized rodents found that the majority of taste-coding neurons were located in deep layers (Yamamoto et al., 1984; Ogawa et al., 1992). More recent work, however, challenged this view, showing that taste responsive neurons can be observed with similar frequency across various layers (Yokota et al., 2011). This recent study also provided evidence that inhibitory interneurons process taste differently from pyramidal cells. Inhibitory neurons had shorter response latencies and longer response durations compared with the pyramidal neurons. Furthermore, they showed narrow taste tuning, in contrast with pyramidal neurons that showed broadly tuned taste responses. However, performing experiments in anesthetized rodents limited the ability to investigate the various patterns of GC activity typically observed in alert animals.

Here, we present results from single unit recordings of putative pyramidal and inhibitory neurons in the superficial and deep layers of GC in alert mice. Activity was recorded with electrode arrays in mice consuming different tastants by actively licking a spout. The behavioral paradigm was optimized for investigating how neurons located in deep and superficial layers encode chemosensory, somatosensory, and anticipatory features related to taste. We found that a large contingent of GC neurons changed their FRs in anticipation of licking initiation and that prestimulus modulations were more frequent for deep relative to superficial layers and for putative inhibitory compared with pyramidal neurons. Firing activity showed rhythmic modulations associated with licking throughout the layers, with a higher phase locking for excitatory cells. With regard to taste coding, we found a larger percentage of chemosensitive neurons in deep layers. While tuning did not show any laminar or cell type difference, decoding performance was higher in pyramidal cells compared with interneurons. Finally, analysis on the convergence of the different types of responses described above revealed that a large portion of neurons throughout the various layers of GC could encode more than one variable.

Altogether, these data demonstrate differences in laminar patterns of chemosensory, somatosensory, and anticipatory activity but also emphasize how all response features are present across the various laminae of GC.

\section{Materials and Methods}

\section{Experimental design}

Experimental subjects

All experimental procedures were performed according to federal, state and university regulations regarding the use of animals in research and approved by the Institutional Animal Care and Use Committee of Stony Brook University. A total of 29 adult female mice (20-25 g; Charles River) were subjects of this study (5 mice for tracing and 24 mice for electrophysiology). We used exclusively female mice to reduce the possible variability associated with sex. Given the low yield of our experimental approach, we chose the focus exclusively on laminar differences in neural activity and did not pursue sex-related differences. Individually housed mice were kept in 12/12 h light/dark cycle and were given ad libitum access to chow and water before the experimental procedures.

\section{Surgical procedures}

Recordings of GC activity in alert, head-restrained mice were performed either chronically by implanting electrodes with a dorsal approach (i.e., lowered from the dorsal surface of the brain) or acutely by inserting electrodes through a lateral window. We chose to use two approaches to optimize yield and accuracy, and to expand our sampling parameters. For both approaches, mice were anesthetized with intraperitoneally injected ketamine/dexdomitor mixture (75 and $1 \mathrm{mg} / \mathrm{kg}$, respectively) and supplemented in small doses as needed (10\% of initial dose). After reaching surgical levels of anesthesia, animals were put on a stereotaxic device. Body temperature was maintained constant $\left(36^{\circ} \mathrm{C}\right)$ throughout the surgery. For chronic implants $(n=11)$ a $1 \mathrm{~mm}^{2}$ craniotomy was performed unilaterally on the dorsal skull surface (stereotaxic coordinates: $1.1 \mathrm{~mm}$ anterior to and $3.3 \mathrm{~mm}$ lateral to bregma). Custom-made, drivable electrode arrays, coated in Di (Invitrogen) were inserted into the brain until the ventral most electrode reached the dorsal boundary of GC (3.1 mm ventral to bregma). The electrode array consisted of 16 wires (formvar coated 0.0015" nichrome microwires, A-M Systems) glued next to each other to form a linear array of $500 \mu \mathrm{m}$ width (Krupa et al., 2004). The tip of the array was cut at an angle (such that electrodes spanned a 500 to $750 \mu \mathrm{m}$ range along the dorsal-ventral axis) and each channel's position within the linear array was mapped. The electrodes were inserted either at 3.5 or at $2.7 \mathrm{~mm}$ lateral to bregma to target superficial or deep layers of GC, respectively. For acute recording preparations targeting GC from the lateral surface of the brain $(n=13)$, the skull overlying the GC was exposed. A recording well was built on the lateral surface using photo-curing bone etchant and dental cement. A thin layer of bone etchant and dental cement were used to cover the exposed skull to prevent tissue growth in the region.

In both chronic and acute preparations, a ground electrode was inserted underneath the skull, above the cerebellum. The microdrive (in chronic surgeries), the ground pin and the head bolt (for restraint) were cemented onto the skull using photo-curing dental cement (Flow-It ALC, Pentron). At the end of the surgeries, animals were injected with Antisedan $(8 \mathrm{mg} / \mathrm{kg})$ for reversal of anesthesia and placed on a heated pad at their home cage for recovery.

Before the first recording sessions in acute preparations (5-10 h prior), animals were anesthetized again using isoflurane (2\%) and a small craniotomy was performed on the lateral surface of the skull to expose the GC. A small tear in the dura was made to facilitate the entry of the linear array. The craniotomy and duratomy were performed above the medial cerebral artery (MCA) and the rhinal vein intersection. This vasculature landmark corresponds to the granular and dysgranular portions of the GC (Chen et al., 2011). Craniotomy was covered with KwikCast, and animals were returned to their home cage until the recording session.

\section{Behavioral training and experimental sessions}

Animals were given one to two weeks to recover from surgery. During training and testing, animals' water intake was limited to $2 \mathrm{ml} / \mathrm{d}$. Animals' weight was kept at $85 \%$ of normal weight for sex, age and strain. Additional water was provided if animals showed signs of dehydration. Mice were first habituated to lick sucrose solution $(0.2 \mathrm{M})$ from a moveable spout while head-restrained. In the subsequent days, water, $\mathrm{NaCl}(0.075 \mathrm{M})$, citric acid $(0.02 \mathrm{M})$, and quinine $(0.001 \mathrm{M})$ solutions (all tastants dissolved in water) were delivered in addition to sucrose. Taste solutions were delivered by a gravity based delivery system, where five independent fluid lines converged in a multibarrel lick spout (Graham et al., 2014). Fluid flow in each line was computer controlled by a separate taste valve such that a $50 \mathrm{~ms}$-long opening of any given valve resulted in formation of a $\sim 2 \mu \mathrm{l}$ droplet. The size of the droplets was calibrated each day before the experiments. After animals habituated to headrestraint and freely licked for all solutions, they were trained on the 
experimental trial structure. Each trial began with an auditory tone ( $2 \mathrm{kHz}$ frequency and $200 \mathrm{~ms}$ in duration), followed (after $1 \mathrm{~s}$ ) by the protraction of the lick spout. The spout contained a preformed drop $(\sim 2 \mu \mathrm{l})$ of solution (one of the five stimuli), and mice had to initiate licking within $1 \mathrm{~s}$ after the spout movement began. For a trial to be considered correct, animals had to do at least six licks at a minimum of $7 \mathrm{~Hz}$ frequency. There was no further delivery of solution after the first drop and animals had to continue licking until the sixth lick delivered a drop of water to rinse the mouth and prepare for the next trial. The spout retracted $1 \mathrm{~s}$ after the rinse. A lick was detected when the animals' tongue crossed the infrared beam positioned right in front of the lick spout. Trials in which animals failed to initiate licking, maintain the required lick rhythm, or do enough licks to obtain a rinse, were considered error trials and were followed by a $20 \mathrm{~s}$ time-out. This trial structure reinforced the natural licking pattern of mice and facilitated obtaining stereotyped licking behavior across tastants, sessions and animals. At the end of each trial, the spout was retracted and cleaned. To clean the lick spout, the water line was opened for $1.5 \mathrm{~s}$ while a motorized computer controlled suction line, positioned just underneath the retracted lick spout, cleaned off the rinsed fluid. The interval between the first licks of two consecutive correct trials was $14.3 \pm 0.02 \mathrm{~s}$. Error trials and trials that were not preceded by a rinse were excluded from the analysis. White noise was played throughout the entire session to obscure any potential auditory cues caused by opening of taste delivery valves. We began recording sessions once animals performed a total of at least one hundred correct trials for three consecutive days.

\section{Electrophysiological recordings}

GC recordings were obtained from head-restrained mice performing the licking task. In mice with chronic implants, linear arrays were lowered by $100 \mu \mathrm{m}$ at the end of each experiment to record from a new subset of neurons in the subsequent session. Daily recording sessions with chronically implanted mice continued until the most dorsal wire reached the ventral boundary of GC determined by stereotaxic coordinates $(4 \mathrm{~mm}$ ventral to bregma). Only recordings from GC, as estimated by the depth of microwires and post hoc histologic analysis of array position, were included in the analysis.

Acute recordings were performed in head-restrained mice after removing the Kwik-Cast cover, and after bathing the exposed brain with cortex buffer ( $125 \mathrm{~mm} \mathrm{NaCl}, 5 \mathrm{~mm} \mathrm{KCl,} 10 \mathrm{~mm}$ glucose, $10 \mathrm{~mm}$ HEPES, $1 \mathrm{M} \mathrm{CaCl}_{2}$, and $1 \mathrm{M} \mathrm{MgSO}_{4} ; \mathrm{pH}$ 7.4). The linear array (same as chronic implants) was coated with Di (Invitrogen) and inserted into GC through the craniotomy on the lateral surface at the intersection of the MCA and the rhinal veins. The array was slowly lowered orthogonal to the surface of GC until the final position was reached (deepest wire at $1000 \mu \mathrm{m}$ ). Upon final positioning of the electrode, the craniotomy was covered with 30,000 cs Dow Corning silicone (Advance Weight Systems Inc.) to ensure stable recordings. The experimental session began $30 \mathrm{~min}$ after the craniotomy was covered. At the end of each acute recording session, the linear array was retracted, the silicone was removed and the craniotomy was covered with Kwik-Cast before returning the animal to its home cage. We limited our acute experiments to two sessions per animal to minimize tissue damage within the recording site.

The two recording methods yielded comparable results in terms of laminar distribution of the recorded neurons (lateral approach: 33 vs 12 , and dorsal approach: 114 vs 64, for deep and superficial layer neurons, respectively; $p=0.2, \chi^{2}$ test). Similarly, comparable was the distribution of neurons' functional properties (lick rhythmic neurons, lateral approach: $16 / 45$, dorsal approach: $83 / 178, p=0.18, \chi^{2}$ test; pre-lick-modulated neurons, lateral approach: $29 / 45$, dorsal approach: $106 / 178, p=0.5, \chi^{2}$ test; chemosensitive neurons, lateral approach: $21 / 45$, dorsal approach: $71 / 178$, $p=0.4, \chi^{2}$ test).

Extracellularly recorded signals were amplified, bandpass filtered $(300-8000 \mathrm{~Hz})$, digitized and recorded (at $40 \mathrm{kHz})$ using Plexon MAP system. Single units were isolated using Offline Sorter software (Plexon). Clustering of waveform features in principal component space as well as analysis of interspike interval histograms were used to isolate action potentials recorded from a single unit (Samuelsen et al., 2012; Jezzini et al., 2013; Gardner and Fontanini, 2014; Liu and Fontanini, 2015). Data were analyzed using custom-written software in MATLAB (The MathWorks). All descriptive statistics are reported as mean \pm SEM unless otherwise specified.

\section{Layer assignments}

In the chronic recording configuration, we used channel mapping, histology and recording depth (along the dorsal-ventral axis) to triangulate the position of each electrode wire. Single units isolated from microwires positioned within the first $350 \mu \mathrm{m}$ of the cortical surface were classified as superficial layer recordings, and units isolated within the $350-850 \mu \mathrm{m}$ depth from the cortical surface were classified as deep layer recordings. In acute recording configurations, where linear arrays were inserted orthogonally to the cortical surface, we used the micromanipulator readout, combined with channel mapping to determine the depth (along medial-lateral axis) of each microwire. The deep and superficial layer assignments were based on the same cortical depth criteria as described above for the chronic recording configuration. Single units recorded outside of GC as indicated by depth of microwire or histologic sections were excluded from analysis.

\section{Cell type assignments}

Single units were classified as putative excitatory or putative inhibitory neurons based on the width of the extracellularly recorded waveforms as described previously in the literature (Cardin et al., 2007; Niell and Stryker, 2008). The width of the waveform was measured as the interval from the trough to the peak (in microseconds). MATLAB's built-in $k$ means clustering algorithm was used to classify neurons into two distinct categories based on the width of the waveforms. This method partitions the data into $k$ clusters by minimizing the sum of all data points' distance to their corresponding cluster center. Histogram of spike width values of the resulting clusters revealed bimodal distribution (bimodality tested with Hartigan's dip test, $p<0.01$ ).

\section{Retrograde tracer injections}

In five mice, $100 \mathrm{nl}$ of cholera-toxin subunit B conjugated to Alexa Fluor 555 (CTB 555), (Invitrogen) was injected unilaterally into GC (stereotaxic coordinates from bregma; AP: $+1 \mathrm{~mm}$, ML: $3.94 \mathrm{~mm}$, DV: -2.4 $\mathrm{mm}$ ). A sharp glass pipette was pulled (Sutter Instruments) the tip was broken, filled with mineral oil, and back filled with $500 \mathrm{nl}$ of CTB 555 . The glass pipette was inserted into Nanoject (Drummond Scientific), and slowly lowered into GC using a micromanipulator (Narishige). A total of 20 puffs of $\sim 5 \mathrm{nl}$ solution were delivered into the region. After the final puff, we waited $15 \mathrm{~min}$ to minimize the spread of the toxin to more dorsal areas before slowly retracting the pipette. The skin was sutured closed and the animals were returned to their home cage to recover. Brains were collected two weeks after the tracer injection.

\section{Histologic procedures}

At the end of experiments, mice were administered a lethal dose of ketamine/dexdomitor mixture and transcardially perfused using PBS followed by $4 \%$ paraformaldehyde (PFA) dissolved in PBS. Brains were removed and postfixed in $4 \%$ PFA overnight; 50 to $100 \mu \mathrm{m}$-thick coronal sections were cut using a Leica vibratome. Brain slices were mounted with media containing fluorescent DAPI to visualize cell nuclei (Fluoromount, Electron Microscopy Sciences). Standard confocal microcopy procedures (Olympus) were used to image the coronal sections and identify the anatomic location of the electrode track as marked by $\mathrm{Di}$ stain.

\section{Statistical analysis}

\section{Area under the curve normalization}

For visualization and analysis of neural activity, FRs of single neurons were aligned to various behavioral events, averaged across trials and normalized using the non-parametric area under the receiver operating curve (auROC) method (Cohen et al., 2012; Gardner and Fontanini, 2014; Liu and Fontanini, 2015). Details on how to implement this method can be found in (Cohen et al., 2012). Briefly, stimulus-evoked FRs for each neuron are normalized to range between 0 and 1 , where 0.5 represents baseline firing (where baseline is defined as the $1 \mathrm{~s}$ interval 
before the onset of auditory tone). Excitatory deviations from baseline activity are valued as $>0.5$, and inhibitory deviations from baseline activity are valued as $<0.5$.

\section{Pre-lick modulation analysis}

For each neuron, trials were aligned to the onset of the first lick. The 500 ms interval before the first lick was divided into two $250 \mathrm{~ms}$ bins. Distribution of FRs obtained from each prestimulus bin was compared with FRs obtained from baseline ( $1 \mathrm{~s}$ baseline before the onset of the cue, divided into $250 \mathrm{~ms}$ bins) using a two-sided, unbalanced MannWhitney $U$ test at $p<0.05$ with Dunn-Sidak correction. Neurons with significantly higher or lower FRs than baseline in at least one prelick bin were classified as pre-lick-excited or -inhibited, respectively. Repeating the analysis with different bin sizes yielded similar results. To assess whether pre-lick modulations were driven by spout movement or lick-related movement, we generated two peristimulus time histograms (PSTH) for each pre-lick-excited neuron: one PSTH aligned to the onset of the spout movement and one PSTH aligned to the onset of the first lick (as shown in Fig. 5A,B, respectively). We limited our analysis to prelick-excited neurons as increases in FR are more robust to analysis than decreases. For each PSTH, we only included spikes that occurred in the interval between the spout onset and the first lick. This ensured that we included the same spike count in each trial for both PSTHs and that the only differences we observed were because of alignment rather than spike count. We then computed the maximum FR of each PSTH and compared the spout onset aligned versus first lick aligned maximum FRs on a scatter plot. In a complementary analysis, we quantified the distance of each spike in the spout-first lick interval to both the first lick and the spout offset. We reasoned that if spikes in this interval are timed closer to the lick onset rather than the spout offset (as shown by smaller distance between spikes and the event), it would indicate that prestimulus excitation is driven by first lick rather than the spout movement. The distribution of spike distances to either events (in absolute values) were compared across all prestimulus excited neurons as shown in Figure $5 C$, right panel ( $p<0.01$, Kolmogorov-Smirnov test).

\section{Mouth movement (MM) analysis}

Orofacial movements were recorded for $42 / 80$ experimental sessions (124/223 units; at 30 frames/s rate). Video, neural recordings, and time stamps for behavioral events were synchronized using Cineplex software (Plexon). Videos were analyzed using custom-written software in MATLAB (Gardner and Fontanini, 2014; Vincis and Fontanini, 2016). The orofacial region was cropped (as shown in Fig. 6A, left panel) and MM analysis was performed on this selected region. For each trial, the MM signal was extracted as the absolute difference in pixel intensity between consecutive frames. For each session, the MM signal intensity was normalized to range between 0 and 100. For 124 neurons recorded in 42 sessions, cross-correlations between $\mathrm{MM}$ and spiking activity (using $33 \mathrm{~ms}$ bin size to match MM sampling rate, lags calculated for 10 bins) were computed on mean subtracted signals during the $3 \mathrm{~s}$ intertrial interval ( $3 \mathrm{~s}$ interval before the auditory tone onset) on a trial by trial basis. Cross-correlations were then averaged across trials and the area under the resulting cross-correlation curve (auXCC) was computed. The significance of cross-correlations was assessed (at $\alpha=0.05$ ) by comparing the auXCC of session average to a null distribution of auXCC created by shuffling the trials of the session (1000 simulated sessions, matching trial count, reshuffled trial order). For neurons with significant crosscorrelation, we determined which signal was the preceding signal by comparing the area under the curve to the left versus the right of the lag $=0$ point. The significance of $(($ LeftauXCC $)-($ RightauXCC $))$ value was assessed by comparing it to the null distribution of the same parameter obtained by shuffling trials as described above.

\section{Change point analysis}

To assess the onset of pre-lick modulations, all trials were aligned to the first lick, and the $500 \mathrm{~ms}$ interval before the lick onset was tested for a significant change point. The first change point in this $500 \mathrm{~ms}$ interval was deemed the onset of prestimulus modulation. Details for this method can be found in (Gallistel et al., 2004; Jezzini et al., 2013).
Briefly, the change point method computes the cumulative distribution function $(\mathrm{CDF})$ of spike occurrences over the baseline (1 s baseline before auditory cue) and prestimulus epoch. The time point in which the slope of the CDF in the pre-lick epoch exceeds the slope of the CDF obtained from the baseline epoch, is marked as a change point.

\section{Stimulus-evoked modulation analysis}

Trials were aligned to the onset of the first lick and the first $500 \mathrm{~ms}$ following the lick onset were divided into two $250 \mathrm{~ms}$ bins. We limited our analysis to the first $500 \mathrm{~ms}$ window after the onset of the first lick as the rinse delivery could occur as early as $500 \mathrm{~ms}$ after the first lick. Only trials that satisfied the behavioral criteria were analyzed. Stimulus-evoked FRs were compared with baseline FRs ( $1 \mathrm{~s}$ baseline before the auditory cue) using two-sided, unbalanced Mann-Whitney $U$ test at $p<0.05$ with Dunn-Sidak correction. Neurons with significantly higher or lower stimulus-evoked FRs than baseline were classified as stimulus-excited or -inhibited, respectively. For stimulus-responsive neurons, the time for return to baseline firing was defined as the first bin within a set of three consecutive bins that were not significantly different compared with baseline FRs (two-sided unbalanced Mann-Whitney $U$ test with DunnSidak correction).

\section{Rhythmic modulation of FR in stimulus-evoked epoch}

For each spike that occurred within the first seven lick cycles, the relative position of the spike with respect to its corresponding lick cycle was computed in radians (for details, see below, Spike-phase consistency). The power spectrum density was computed for the lick-warped PSTHs using MATLAB's built-in periodogram function using 2 to $20 \mathrm{~Hz}$ bandpass filter and $\pi / 60$ radian bin size (corresponds to $\sim 1.1 \mathrm{~ms}$ ). Power spectra were normalized so that the sum of the power was 100 . A neuron's FR pattern was classified as rhythmically modulated if the neuron's power spectrum had a peak in the 6 to $10 \mathrm{~Hz}$ range and if the amplitude of the peak was at least 2 SDs greater than the median value of the signal.

\section{Spike-phase consistency}

For each spike that occurred within the first seven lick cycles, the relative position of the spike with respect to its corresponding lick cycle was computed in radians using the following formula:

$$
(2 \pi) *\left(\frac{\text { SpikeTime }- \text { LickBegin }}{\text { LickCycle }}\right) .
$$

The variables spike time, lick begin, and lick cycle were computed in seconds. Lick cycle was computed as the duration between two consecutive licks. Converting spike times from seconds to radians allowed us to quantify the relative position of the spike with respect to the lick cycle and compare across trials, sessions and animals. After all the spike times were converted to radians, the $0 \pi-2 \pi$ interval was then divided into eight phase bins. On average $2 \pi$ radians corresponded to $135 \mathrm{~ms}$, therefore each phase-bin was roughly equivalent to $17 \mathrm{~ms}$. We computed the probability of observing a spike in a particular phase-bin given the neuron's total spike count for all the phase-bins. We then compared this probability distribution to a uniform probability distribution (i.e., the case in which the probability of observing a spike is the same across all phase-bins of the lick cycle) given by (1/number of bins). The difference between the observed and the uniform probability distributions was quantified using total variation distance and standardized by dividing by the number of trials. Spike-phase consistency measure across cell categories was compared using Kruskal-Wallis test followed by multiple comparisons using Bonferroni correction. Varying the bin size did not alter the results across four different bin sizes we tested.

Taste coding analysis

Linear support vector machine (SVM) classifiers were implemented in MATLAB using the software's built-in Classification toolbox (fitcecoc.m) and custom scripts. For single unit decoding, $z$-scored spike counts from the first five lick-bins of single trials (lick-warped data from first lick to the delivery of rinse computed as described above) were used to train the classifier. The performance of the trained classifier was computed using 


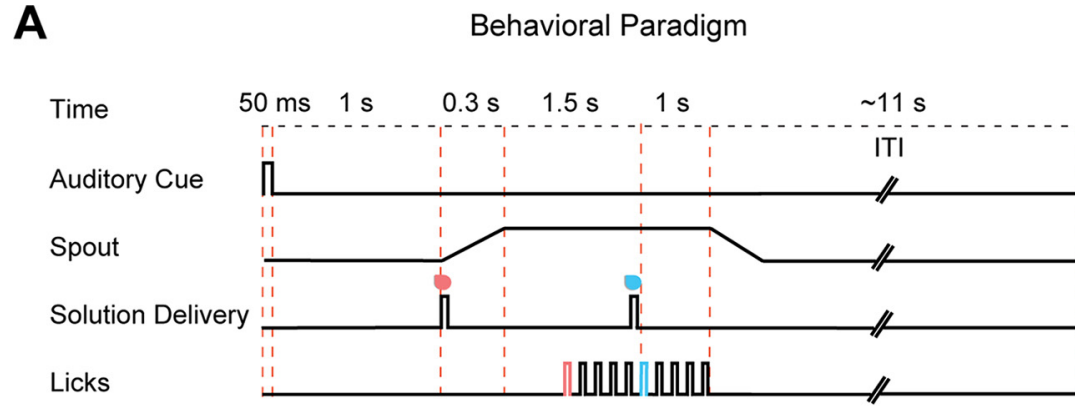

\section{B Licking Behavior}

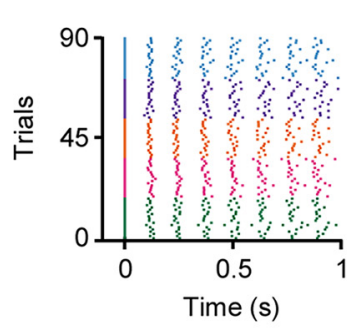

C

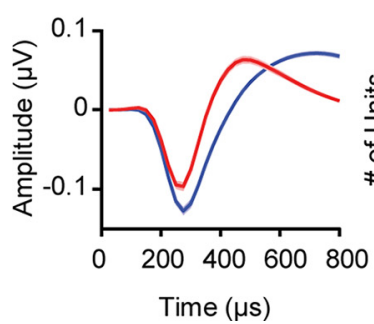

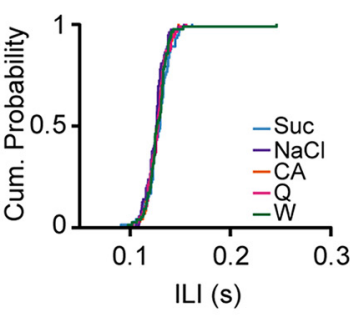

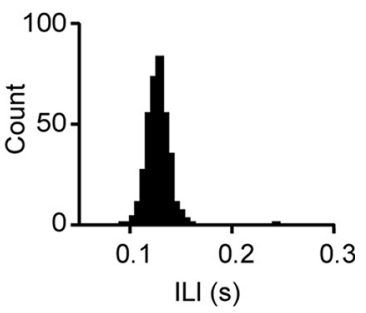

Cell Type Classification
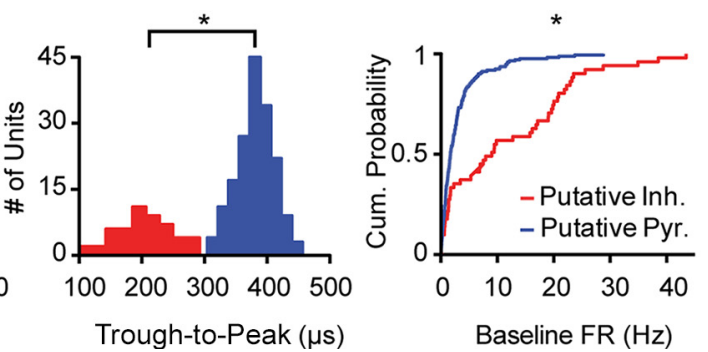

Figure 1. Behavioral paradigm and waveform analysis. $\boldsymbol{A}$, Schematic representation of the behavioral paradigm. $\boldsymbol{B}$, Representative licking behavior from a single session. Left panel, Licking behavior for all five stimuli across a session. Trials are sorted by stimulus type for visualization (color-stimulus association same as in the middle panel). Each row is a trial and each dot represents a lick, all trials are aligned to the first lick. Only the first seven licks of each trial are shown. Middle panel, Cumulative distribution function of the interlick interval (ILI) for each type of taste trial. Right panel, Distribution of ILI's for all trials in the session. C, Classifying cell types based on action potential waveform. Left, Average waveforms for putative inhibitory neurons (in red) and putative pyramidal cells (in blue). Middle, Distribution of action potential widths for putative inhibitory (in red) and putative pyramidal (in blue) neurons. Right, Cumulative distribution function of baseline FRs of putative inhibitory and putative pyramidal neurons. Asterisks indicate significant differences, $p<0.01$ (Hartigan's dip test for the middle panel; KolmogorovSmirnov goodness of fit test for the right panel).

leave-one-out cross-validation method. Decoding performance for a given taste stimulus was defined as the percentage of correctly classified test trials over all trials for that taste. A neuron's activity was said to successfully decode a specific tastant if the confusion matrix satisfied the following criteria: (1) the decoding performance for the tastant was above chance level and the trials of that tastant were not misclassified more frequently as another tastant (i.e., if sucrose trials were correctly classified as sucrose $30 \%$ of the time, but were misclassified as salt $40 \%$ of the time, this was not considered successful decoding for sucrose condition); (2) another tastant was not misclassified as a particular tastant more frequently than the actual tastant itself (i.e., if $30 \%$ of the trials predicted as sucrose were actually sucrose but $40 \%$ of trials predicted as sucrose were salt trials, this was not considered successful decoding of sucrose). We repeated the decoding analysis using both regular PSTH (duration and bin-size matched for lick-warped data) and lick-warped data with linear discriminant analysis (fitcdiscr.m, MATLAB), and weighted k-nearest neighbor classification algorithms (fitcknn.m, MATLAB) keeping the same cross validation procedure. All methods resulted in qualitatively similar results with no more than $2.2 \%$ difference in the total number of taste-coding neurons. Breadth of tuning of single neurons was determined as the number of tastants that could be decoded successfully. For comparisons of average decoding performance across categories, only the values from successfully decoded tastants were included in the mean computations. For time-resolved decoding analysis, we applied the same decoding method as described above (SVM classifier with leave-one-out cross-validation method) on single, consecutive bins to resolve the time course of single neuron decoding. For each neuron we computed a lickwarped PSTH until the sixth lick (water drop delivery) and concatenated with a regular PSTH aligned to the sixth lick, binned at $135 \mathrm{~ms}$ (to match the average lick cycle). We used non lick-warped data following the sixth lick as animals would often stop licking shortly after the water drop and lick numbers past the water-lick were variable across trials and animals.

\section{Results}

Single unit activity (223 neurons) was recorded across 80 sessions from 24 head-restrained mice performing a tasting paradigm designed to standardize licking to different taste stimuli. On average we recorded $2.7 \pm 1.7$ neurons per session. Figure $1 \mathrm{~A}$ shows the structure of the paradigm (for details, see Materials and Methods and figure legend). After training, mice displayed stereotyped licking behavior across different stimuli throughout the recording session (mean interlick interval across all tastes $=0.135 \pm 0.035 \mathrm{~s}$; Fig. $1 B$ ). On average mice performed $100 \pm 34$ trials in a session (session duration: $27 \pm 8.2 \mathrm{~min}$.). 
A

Injection Site

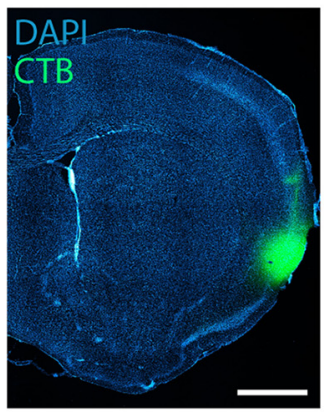

D

Dorsal Implant Deep Layers Targeted

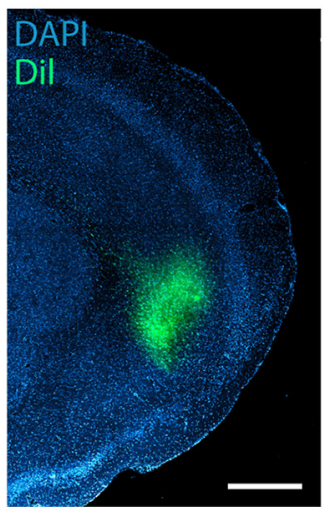

B Retrogradely labeled cell bodies in VPMpc

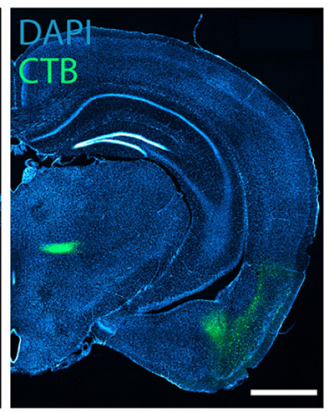

E Dorsal Implant Superficial Layers Targeted
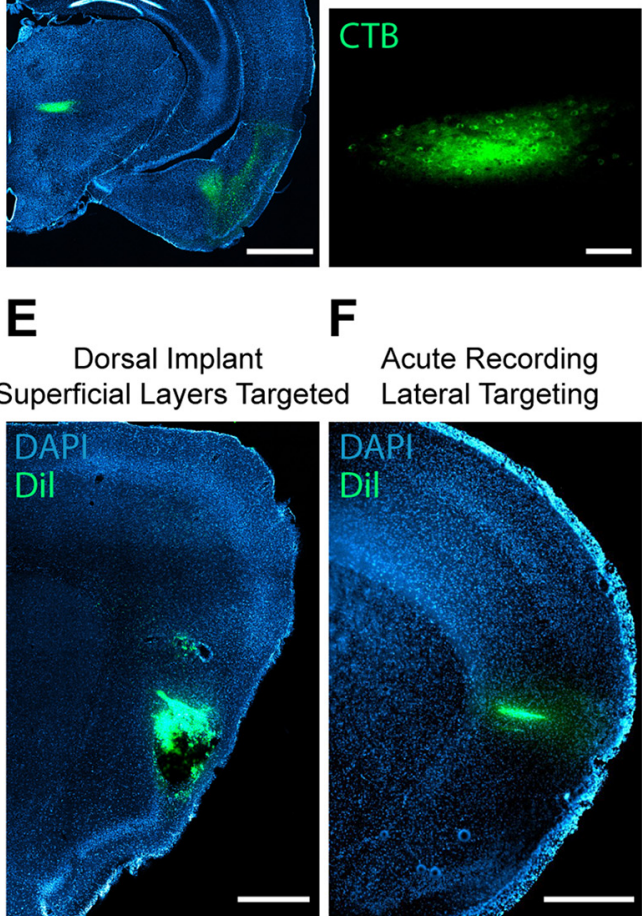

F

Acute Recording Lateral Targeting

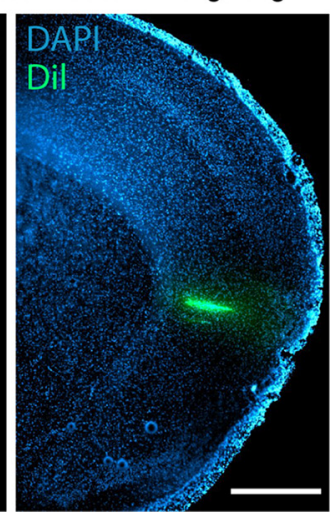

Figure 2. Retrograde tracer injections and recording site confirmation. $\boldsymbol{A}$, Retrograde tracer injection into GC. Coronal section showing retrograde tracer (TB 555 injection site (bregma: $+0.8 \mathrm{~mm}$ ). Nuclear counterstain with DAPI is shown pseudocolored in cyan. (TB 555 is shown in green. Scale bar: $1 \mathrm{~mm}$. B, Retrogradely labeled cell bodies in VPMpc. Coronal section (bregma: $-2.4 \mathrm{~mm}$ ) shows retrogradely filled cell bodies in VPMpc. Scale bar: $1 \mathrm{~mm}$. C, Magnification of retrogradely filled cell bodies in VPMpc in the same coronal section as $\boldsymbol{B}$. Scale bar: $0.1 \mathrm{~mm}$. $\boldsymbol{D}$, Histology from chronic, dorsal electrode implant targeting deep layers of GC (bregma: $+0.9 \mathrm{~mm}$ ). Nuclear counterstain with DAPI (pseudocolored in cyan). Tip of the electrode coated in Dil (shown in green). Scale bar: $0.5 \mathrm{~mm}$. $\boldsymbol{E}$, Histology from chronic, dorsal electrode implant targeting superficial layers of GC (bregma: $+1.0 \mathrm{~mm}$ ). Scale bar: $0.5 \mathrm{~mm}$. $\boldsymbol{F}$, Histology from acute recording configuration, electrodes inserted from the lateral side of the brain, entering orthogonal to layers of cortex (bregma: $+1.0 \mathrm{~mm}$ ). Scale bar: $0.5 \mathrm{~mm}$.

\section{Layer and cell type identification}

Recordings were targeted to the portion of the GC that receives direct input from the gustatory thalamus (VPMpc) as shown by retrograde tracer injections (Fig. $2 A-C$ ). To record firing activity of GC neurons we relied on chronic (Fig. 2D,E) and acute extracellular recordings (Fig. $2 F$ ) from deep and superficial layers of GC in alert mice. Deep and superficial layers were defined based on the relative position of the microwires with respect to the cortical surface (see Materials and Methods, Layer assignments). A total of 223 single units were isolated across animals and sessions, with 147 units recorded from deep layers and 76 units recorded from superficial layers. Neurons were further classified into putative excitatory and inhibitory cell type categories based on the width of extracellularly recorded action potential waveforms (Fig. 1C). The distribution of action potential widths from all isolated single neurons was classified into two nonoverlapping categories using a $k$-means clustering algorithm; $23 \%$ of units $(51 / 223)$ showed narrow waveforms $($ mean $=203.4$, standard deviation $(\mathrm{std})=49.5 \mu \mathrm{s})$ and were classified as putative inhibitory neurons; $77 \%$ of the neurons (172/223) had wide waveforms
$($ mean $=375.9$, std $=101.1 \mu \mathrm{s})$ and were classified as putative excitatory neurons. The distribution of action potential widths showed a bimodal distribution (Hartigan's dip test, $p<0.01$; Fig. $1 C$, middle panel). Furthermore, the population of putative inhibitory neurons showed significantly higher baseline FRs compared with putative excitatory neurons, further confirming the classification $(p<0.0001$, Kolmogorov-Smirnov goodness of fit test; Fig. $1 C$, right panel).

\section{Baseline and evoked FRs of GC neurons are layer and cell type-specific}

Upon classifying neurons based on laminar position and action potential waveform, we created four categories: deep layer pyramidal neurons (DP; 101/223), deep layer inhibitory neurons (DI; 46/223), superficial layer pyramidal neurons (SP; 71/223), and superficial layer inhibitory neurons (SI; 5/223). Box plots of baseline and stimulus-evoked FRs of all four categories of neurons are shown in Figure $3 A$. Baseline FRs were significantly different across categories (DI: $12.98 \pm$ $1.64 \mathrm{~Hz}, \quad \mathrm{DP}: \quad 3.80 \pm 0.51 \mathrm{~Hz}, \quad \mathrm{SP}: \quad 2.0 \pm$ $0.23 \mathrm{~Hz}$, SI: $0.62 \pm 0.43 \mathrm{~Hz}, p<0.0001$, Kruskal-Wallis test) with DI neurons showing greater baseline FRs compared with DP, SP, and SI neurons (for all, $p<0.001$, post hoc multiple comparisons with Bonferroni correction). Baseline FRs did not differ among DP, SP, and SI neurons. Stimulus evoked FRs were also significantly different across categories of neurons (DI: $35.59 \pm$ $5.12 \mathrm{~Hz}, \quad$ DP: $7.07 \pm 1.04 \mathrm{~Hz}, \quad$ SP: $3.23 \pm$ $0.7 \mathrm{~Hz}, \quad$ SI: $\quad 6.66 \pm 3.63 \mathrm{~Hz}, \quad p<0.0001$, Kruskal-Wallis), with DI neurons showing significantly greater FRs compared with DP, SP neurons (for both, $p<0.001$, post hoc multiple comparisons with Bonferroni correction), but not compared with SI neurons. Interestingly, while DP and SP showed similar FR distributions at baseline, DP neurons had significantly higher FRs in the stimulus-evoked period compared with SP neurons $(p=0.01$, post hoc multiple comparisons with Bonferroni correction).

\section{GC neurons display pre-lick modulations}

To visualize the time course of FR modulations for the entire population, each neuron's average firing activity was normalized using auROC normalization and plotted in a single row as a heat map (Fig. 3B). Visual inspection of population activity aligned to the first lick shows that in a subset of neurons FR modulations begin before the animal's tongue contacts the spout. To investigate the pre-lick activity observed in Figure $3 B$, we classified each neuron as either pre-lick modulated or not based on the presence of significant changes from baseline FRs in the $500 \mathrm{~ms}$ interval before the first lick. Overall, 60\% of neurons (135/223) in GC showed pre-lick modulations with 38\% (85/223) showing excitatory and 22\% (50/223) showing inhibitory modulations. On average, the onset of pre-lick modulations occurred $0.20 \pm 0.01 \mathrm{~s}$ 
A Baseline and Stimulus-Evoked Firing Rates

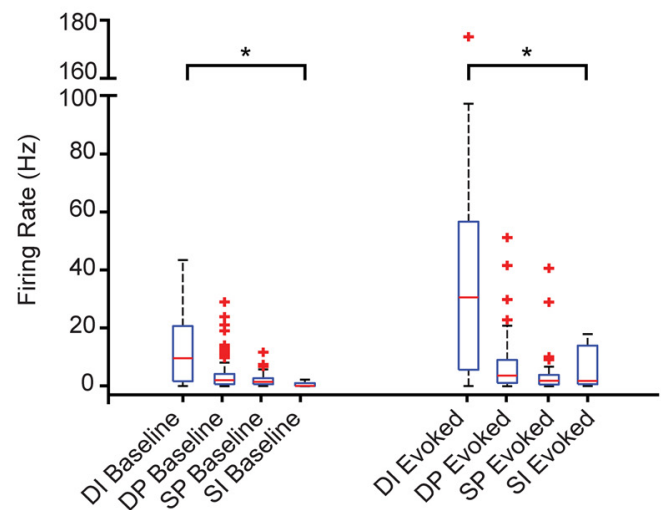

B

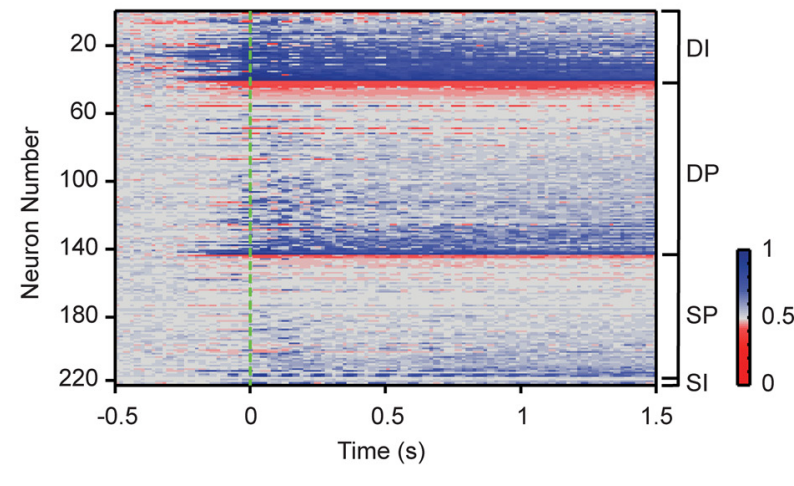

Figure 3. First lick aligned response patterns of GC neurons. A, Box plots for baseline and stimulus-evoked FRs in each category of neurons: deep pyramidal (DP), deep inhibitory (DI), superficial pyramidal (SP), and SI. For a given category, red horizontal lines indicate median value, lower and upper boundaries of the box plot (in blue) represent the 25th and 75th percentile respectively, and red crosses indicate the outliers. $\boldsymbol{B}$, Heat map of auROC normalized population activity aligned to the first lick (time 0 ). Neurons are grouped into categories according to their layer and putative cell type. Each category is sorted in ascending order by their average FR in the stimulus-evoked interval (0-1.5 s). Excitatory responses are shown in blue and inhibitory responses in red. Asterisk indicates $p<0.0001$ for FR differences across all categories (Kruskal-Wallis test).

A
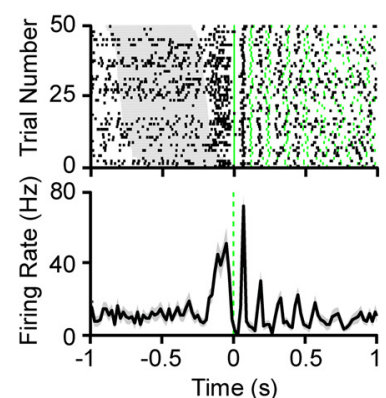

C

Deep Pyramidal

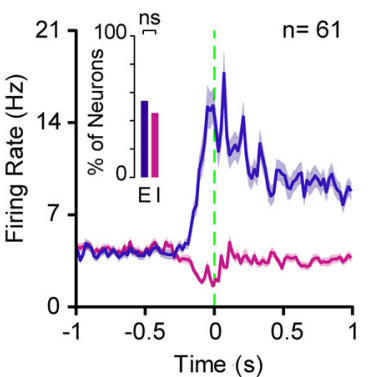

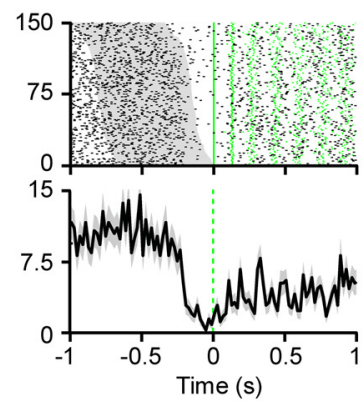

Prestimulus Pop. Response Dynamics Across Categories
B Percentage of Prestimulus Modulated Neurons

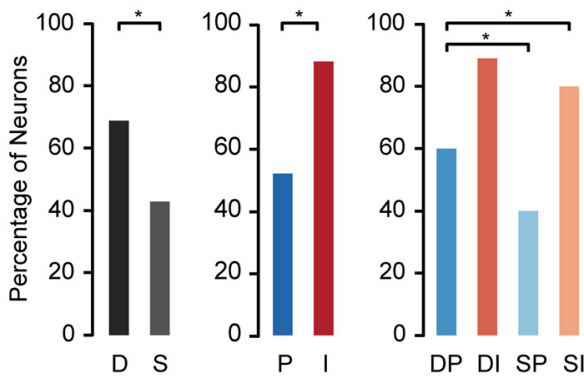

Deep Inhibitory

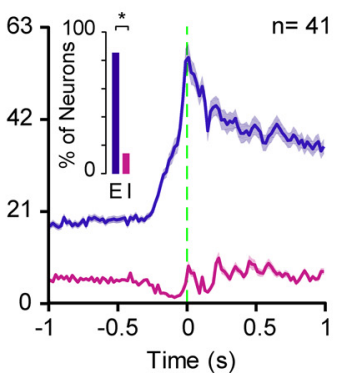

Superficial Pyramidal

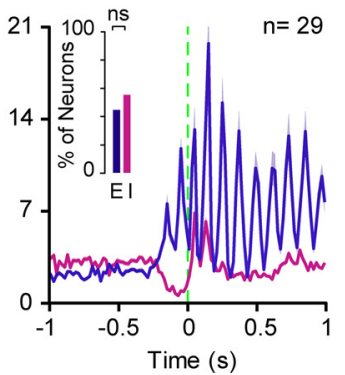

Superficial Inhibitory

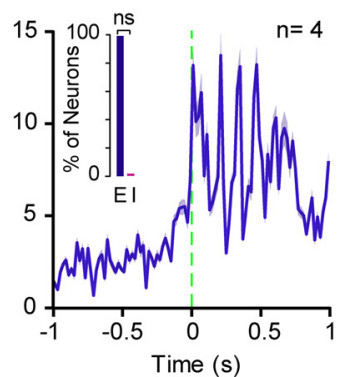

Figure 4. Prestimulus responses in GC. $\boldsymbol{A}$, Representative prestimulus-modulated neurons. Left and right panels, Raster plots (top) and PSTHs (bottom) for a prestimulus-excited and prestimulus-inhibited neuron, respectively. Trials are aligned to the first lick (time 0 ) and sorted in ascending order based on spout offset to first lick time. Gray shaded bars in the raster indicate the duration of spout movement in each trial. Green dots mark the licks. B, Percentage of prestimulus-modulated neurons in each category of neurons. Asterisk indicates $p<0.001, \chi^{2}$ test. $C$, Prestimulus response dynamics across categories. Population PSTH of prestimulus-excited (in purple) and prestimulus-inhibited neurons (in magenta) are shown. Insets show the percentage of excited and inhibited neurons within the prestimulus-modulated category. Significance of relative percentages tested using binomial test, asterisk indicates $p<0.0001$, ns indicates $p>0.05$.

before the first lick, with no category-specific differences in modulation onset (DP: $-0.18 \pm 0.02$ s, DI: $-0.21 \pm 0.03$ s, SP: $-0.20 \pm 0.02 \mathrm{~s}$, SI: $-0.17 \pm 0.09 \mathrm{~s}, p=0.58$, Kruskal-Wallis test). Figure $4 \mathrm{~A}$ shows raster plots and PSTHs for two representative neurons that display excitatory (left) and inhibitory (right) FR changes before the first lick. The relative proportions of prestimulus-modulated neurons were significantly different across the four categories of neurons (DP: 60\%, 61/101, DI: 89\%, 41/46, SP: $41 \%, 29 / 71$, SI: $80 \%, 4 / 5),\left(p<0.0001, \chi^{2}\right.$ test; Fig. $\left.4 B\right)$. A greater proportion of deep layer neurons $(69 \%, 102 / 147)$ displayed prestimulus modulations compared with superficial layer neurons (43\%, 33/76; $p<0.001, \chi^{2}$ test), so was the case for inhibitory neurons $(88 \%, 45 / 51)$ compared with pyramidal neurons $(52 \%$, 90/172; $p<0.0001, \chi^{2}$ test). Next, we investigated whether the 
A
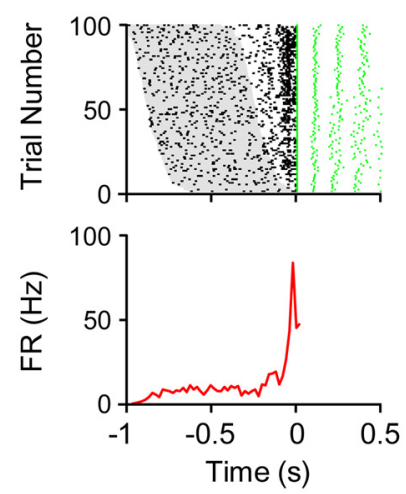

B Spout Onset Aligned

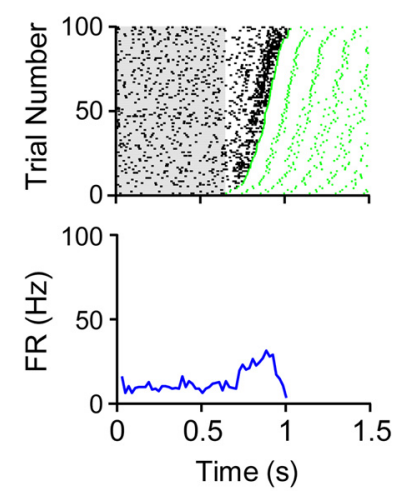

C Alignment of Prestimulus Modulation

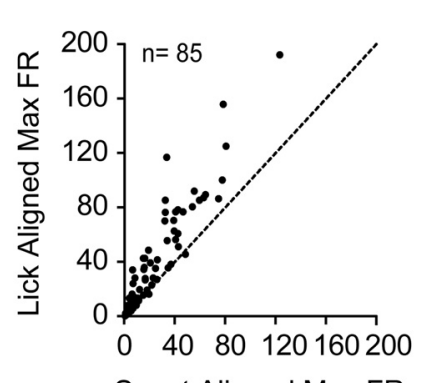

Spout Aligned Max FR

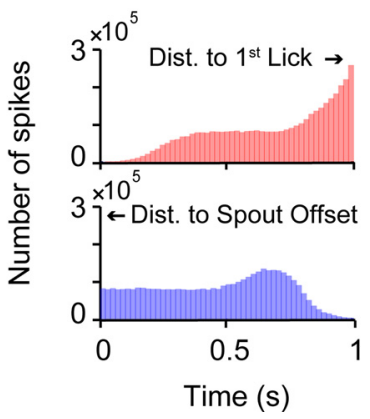

Figure 5. Prestimulus modulation is aligned to first lick. $\boldsymbol{A}$, Raster plot of a representative prestimulus-excited neuron. Trials are aligned to the first lick at time 0 . Gray shaded bars represent the duration of the spout movement for each trial. Green dots represent licks. Trials are sorted in ascending order according to the distance from spout offset to first lick. Only spikes that occur within the interval between the spout onset and first lick are plotted for visualizing alignment of spike times. $\boldsymbol{B}$, Raster plot and PSTH of same neuron as in $\boldsymbol{A}$, with spikes aligned to the spout onset at time 0 . $\boldsymbol{C}$, left panel, Scatter plot of maximum FRs in first lick aligned versus spout-onset aligned PSTHs. Each data point represents the session average of one prestimulus-excited neuron. Right panel, Histogram of spike distances from all prestimulus-excited neurons to first lick (top panel) when spikes are aligned to first lick (first lick at $t=1$ ) and histogram of spike distances to spout offset (bottom panel) when spikes are aligned to spike offset (spout offset at $t=0$ ).

proportion of neurons excited or inhibited in the pre-lick period differed across layers and cell types (Fig. 4C, insets). DP and SP displayed a similar percentage of neurons that were either excited or inhibited in the pre-lick period $(p>0.5$ for both, binomial test, DP excitatory $54 \%, 33 / 61$, inhibitory: $46 \%, 28 / 61$; SP, excitatory: $45 \%, 13 / 29$, inhibitory: $55 \%, 16 / 29)$. On the contrary, a larger proportion of DI neurons showed excitatory modulations during the pre-lick phase compared with inhibitory modulations $(p<0.0001$, binomial test, excitatory modulations: $85 \%, 35 / 41$ vs inhibitory modulations: $15 \%, 6 / 41)$. The few SI neurons recorded (prestimulus-modulated neurons, 4/5) were exclusively excited in the pre-lick phase (4/4). Figure $4 C$ features population-PSTHs for the different groups of pre-lick-modulated neuron (DP, DI, SP, and SI, either excited or inhibited).

Altogether these results show that GC neurons display robust FR modulations in the pre-lick epoch starting on average $200 \mathrm{~ms}$ before the first lick and these modulations occur predominantly in DI neurons.

Timing of pre-lick modulations and relationship with MMs Pre-lick changes in FRs could be promoted by different behavioral events. First, we addressed the possibility that pre-lick modulations might be associated with the movement of the spout cueing the animal for the imminent stimulus. The interval between the onset of the spout movement and the initiation of the first lick was variable across trials $(0.76 \pm 0.12 \mathrm{~s})$, as mice could initiate licking at any time within the $1 \mathrm{~s}$ following the beginning of the spout's protrusion. We exploited this variability to assess whether pre-lick modulations were better aligned to the spout movement or to the time of the first lick. We computed the spiking activity for pre-lick modulated neurons by aligning the timing of action potentials either to the first lick or to the spout movement onset. Figure 5 shows raster plots of one representative, prestimulus excited neuron aligned to the first lick (Fig. 5A) and to the spout movement onset (Fig. 5B). Visual inspection reveals that prestimulus excitation is locked to the onset of the first lick. PSTHs corresponding to these raster plots (Fig. $5 A, B$, bottom panel) confirm this effect, showing that the peak FR is higher in the first lick aligned PSTH (peak FR $=86.2 \mathrm{~Hz}$ ) compared with the spout onset aligned PSTH (peak FR $=32.1 \mathrm{~Hz}$ ). The scatter plot in Figure 5C shows that, for 83 out of 85 prestimulus-excited neurons, lickaligned PSTHs yield higher peak values compared with spout-aligned PSTHs. In a second complementary analysis we computed the distance of each spike (in the time interval from spout offset to first lick) to both the spout offset and the first lick. We pooled spike distances for the two events (distance to first lick and distance to spout offset) across all prestimulus excited neurons. As shown in Figure $5 C$, right panel, we observed that the spike density of the population increases before the first lick. This pattern differed from the profile of spike density for the spout offset condition, where the spike density peaks much further in time and the peak itself is smaller compared with the lick onset condition (KolmogorovSmirnov test, $p<0.01$ ). Altogether, these results suggest that prestimulus activity is not triggered by the spout movement as a cue but rather is aligned to the first lick.

Next, we performed a series of analyses aimed at understanding the relationship between prestimulus modulations and MMs. It has been shown that, in addition to MMs during consummatory behavior, mice can move their mouth spontaneously or in anticipation of expected fluid (Gardner and Fontanini, 2014; Vincis and Fontanini, 2016). We analyzed video recordings of the orofacial region for sessions covering 124/223 units. As shown in the representative examples in Figure 6A, traces obtained from the analysis of MMs (middle trace) captured licking activity (top trace). In addition to large licking movements, video analysis captured small MMs that occurred when the spout (along with the lickometer) was in its retracted position. Upon validating the video analysis, we compared the time course of prestimulus-modulated population activity with that of mouthmovement activity. Figure $6 B$ shows the population average of prestimulus-excited (in blue) and inhibited (in red) neurons 
overlaid with average of MM signals recorded from the corresponding sessions (in black). Visual inspection of the traces suggests that the average latency of prestimulus activity may, on average, precede the onset of MMs. To quantify the correlation between MMs and GC neural activity on a cell-by-cell basis, and to assess the temporal relationship between the two signals, we performed a trial-by-trial crosscorrelation analysis. For this analysis, we focused on baseline spiking activity and MMs. This analysis revealed that $63 \%$ (51/ 82) of the prestimulus-modulated neurons show significant cross-correlations with MMs (Fig. 6C, left panel). Neurons with significant cross-correlations display both positive $(31 \%, 25 / 82)$ and negative $(32 \%$, 26/82) correlations with MMs. Next, we quantified the temporal relationship between MM and neural activity in prestimulus-modulated neurons with significant cross-correlations. Figure $6 C$, middle panel, shows the heat map of cross-correlation. This plot shows that peaks occur at widely ranging lag points. Classification of cross-correlations according to where the peak occurs, shows that 57\% (29/51) of neurons display broad cross-correlation peaks around lag $=0,33 \%(17 / 51)$ of neurons show spiking activity preceding $\mathrm{MMs}$, and lastly $10 \%(5 / 51)$ of neurons show MMs preceding neural activity. Hence, the majority of neurons with correlations to mouth movement showed broad cross-correlations where neither one of the signals was clearly preceding the other. When a signal was preceding another, it was more frequently the neural activity preceding the MMs.

Across laminar and cell type categories, there were no differences in the proportion of neurons that showed significant (DP: 26/42, DI: 18/27, SP: 7/12) and non-significant correlations (DP: 16/42, DI: $9 / 27$, SP: 5/12) with mouth movements $\left(p=0.87, \chi^{2}\right.$ test). Furthermore, among the neurons that were correlated with mouth-movements, there were no consistent trends in the temporal relationship between of FR and MM signals $\left(p=0.28, \chi^{2}\right.$ test; broad correlations: DP:16/26, DI:9/18, SP: 4/7, MM preceding: DP: 3/26, DI:0/18, SP: 2/7, or FR preceding: DP: 7/26, DI: 9/ 18, SP: $1 / 7)$.

Overall, these results demonstrate that not all prestimulusmodulated neurons are correlated with MMs. More importantly, in the population of neurons whose activity is significantly correlated with MM, the temporal relationship between spiking and movement is widely varying. These results suggest that prestimulus modulations cannot simply be accounted by mouth movements acting as a sensory signal and driving the neural activity in GC.

\section{Licking-related activity}

Besides being activated in anticipation of licking, GC neurons are known to be engaged by licking and tasting. Neurons were classified as stimulus responsive based on the presence of significant changes in FRs relative to baseline in the stimulus epoch. The great majority $(88 \%, 197 / 223)$ of neurons in GC were responsive within the first $500 \mathrm{~ms}$ from the first lick. Deep layer neurons showed a greater percentage $(94 \%, 138 / 147)$ of stimulus responsive neurons compared with superficial layer neurons $(78 \%, 59 / 76)$ and inhibitory neurons showed greater percentage of stimulus responsive neurons $(96 \%, 49 / 51)$ compared with pyramidal neurons $\left(86 \%, 148 / 172\right.$; both $p<0.05, \chi^{2}$ test). Across all four categories, DI neurons showed the highest percentage $98 \%$ (45/46), with SP neurons showing the lowest percentage $(77 \%, 55 / 71)$ of stimulus responsive neurons $\left(p<0.01, \chi^{2}\right.$ test). Across all cell categories, a greater percentage of stimulus responses were excitatory (DP: 77\%, DI: 87\%, SP: 60\%, SI: 100\%) compared with inhibitory (DP: $23 \%$, DI: $13 \%$, SP: $40 \%$, SI: $0 \%$ ). The relative distribution of excitatory versus inhibitory stimulus modulations was significantly different across categories $(p<$ $0.05, \chi^{2}$ test) with SP neurons showing higher inhibitory stimulus modulations compared with the other cell categories. Next, we computed the time it took to return to baseline FRs for all stimulus responsive neurons. The return to baseline times for the four categories of neurons were significantly different from one another (DI: $2.77 \pm 1.7$ s, DP: $2.16 \pm 1.8 \mathrm{~s}$, SP: $1.89 \pm 1.7 \mathrm{~s}$, SI: $3.5 \pm 1.2 \mathrm{~s}, p<0.01$, Kruskal-Wallis test), with DI neurons showing significantly slower return times compared with SP neurons $(p<0.01$, post hoc multiple comparisons with Bonferroni correction). Population PSTHs of stimulus excited neurons (Fig. $7 A$ ) revealed rhythmic firing patterns during the stimulus period. We analyzed rhythmicity at the single neuron level by computing the power spectrum within the $1 \mathrm{~s}$ interval following the first lick in lick-warped PSTHs (see Materials and Methods, Rhythmic modulation of FR in stimulus-evoked epoch). Using this criterion, $44 \%$ of neurons in GC (99/223) showed rhythmic activity in the stimulus epoch. The prevalence of rhythmic activity was 


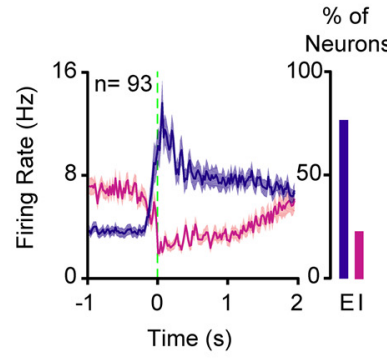

B

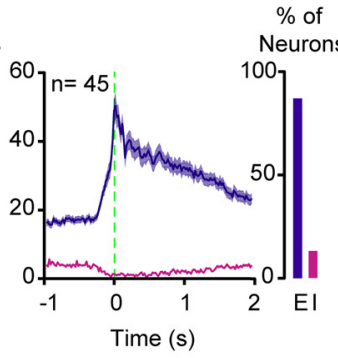

Lick Cycle Aligned Spike Times
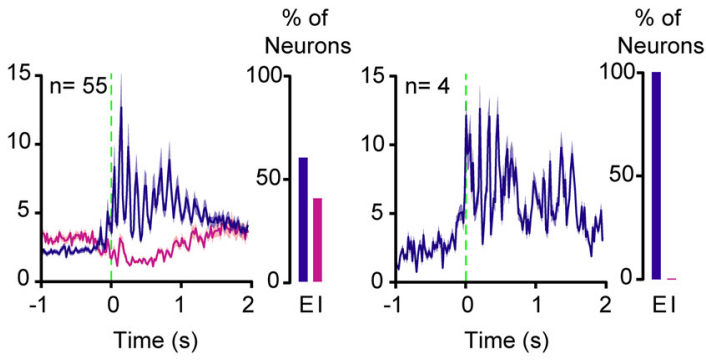

C Spike-Phase Specificity
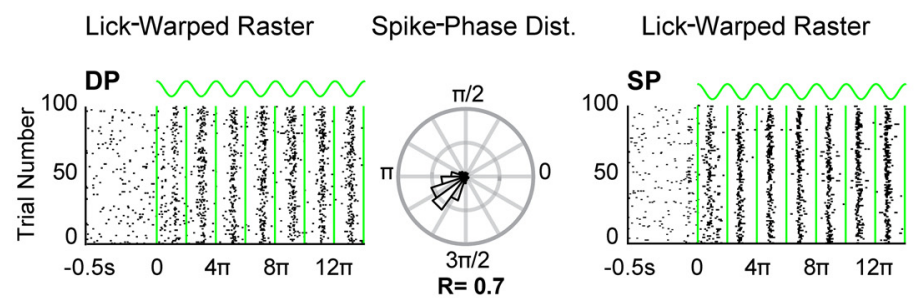

Spike-Phase Dist.
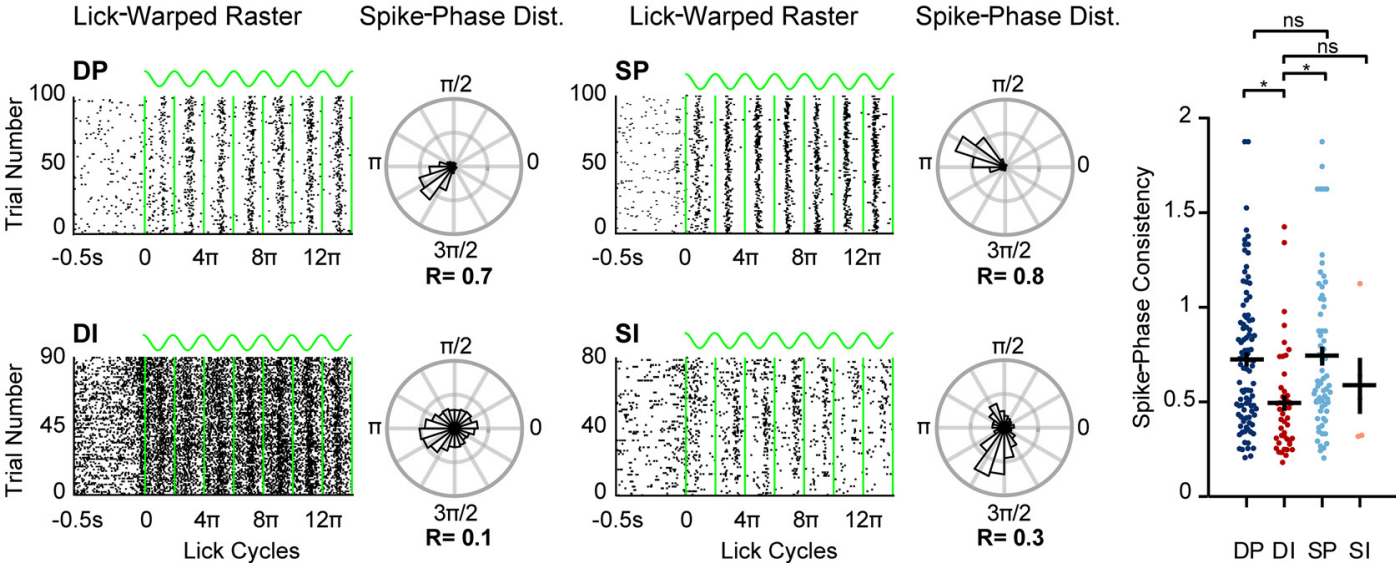

Figure 7. Stimulus-evoked and licking-related activity of $\mathrm{GC}$ neurons. $A$, Stimulus-evoked response dynamics across categories. For each category, population PSTHs aligned to the first lick (at time $=0$ ) of stimulus-excited (in purple) and stimulus-inhibited neurons (in magenta) are shown. Bar graphs show the relative percentage of stimulus excited and stimulus inhibited neurons for each cell category. $\boldsymbol{B}$, Representative neural activity of four neurons with respect to lick cycles. For each neuron, the left plot shows the baseline activity before the first lick ( -0.5 to 0 s) concatenated with lick-warped raster plots, where the relative position of spikes within the first seven lick cycles are plotted in radians. Vertical green lines represent the beginning and end of the lick cycles. The last column shows the phase distribution of all spikes that occur within the first seven lick cycles on a rose plot. The $R$ value next to the rose plot shows the mean vector length of the circular distribution. C, Spike-phase consistency distributions for each cell category. Mean (horizontal black line) and SEM (vertical black line) of spike-phase consistency is shown for each category of neurons. The group means are compared using Kruskal-Wallis test followed with multiple comparisons using Bonferroni corrections (asterisk indicates $p<0.001$ and ns indicates $p>0.05$ ).

different across cell categories where 53\% (54/101) of DP neurons, $28 \%$ (13/46) DI neurons, 44\% (31/71) of SP neurons and 20\% (1/ $5)$ of SI neurons showed rhythmic activity $\left(p<0.05, \chi^{2}\right.$ test).

We created lick-warped raster plots to study the phase relationship between spike timing and licking. For each neuron, we computed the position of spikes with respect to the lick cycle they occurred in (see Materials and Methods, Spike-phase consistency). Figure $7 B$ shows four representative neurons whose firing revealed rhythmicity. Lick-warped raster plots of representative DP and SP neurons illustrate the case in which spikes in the stimulus epoch occur consistently at a specific phase of the lick cycle across consecutive licks and trials. The corresponding rose plots of DP and SP neurons confirm this observation. In contrast, for the representative SI and DI neurons, spikes are distributed more broadly across the possible phase space. For each neuron, we quantified the extent to which spikes occur preferentially at specific phases of the lick cycle by using the spike-phase consistency measure (see Materials and Methods, Spike-phase consistency). Using this method, neurons whose spikes are positioned uniformly across the lick cycle have small spike-phase consistency values, whereas neurons whose spikes cluster at particular phases of the lick cycle have high spike-phase consistency values. Figure $7 C$ displays the spike-phase consistency distributions for each cell category. We found that spike-phase consistency is different across cell categories (DP: $0.73 \pm 0.04$, DI: $0.49 \pm 0.04$, SP: $0.74 \pm 0.05$, SI: $0.58 \pm 0.15, p<0.001$, Kruskal-Wallis test). Post hoc multiple comparisons across categories revealed that both DP and SP neurons show significantly higher spike-phase consistency compared with DI neurons (for both; $p<0.001$, post hoc multiple comparisons with Bonferroni correction) while the DP and SP neurons are not significantly different from each other. To assess whether spike timing precision varies as a function of FR we correlated the spike-phase consistency measures with the total spike count (both computed for the same time window of five lick cycles, $R=-0.27, p<$ $0.001)$. The significant correlation suggests that the firing frequency of the different categories may also play a role in determining the spike timing precision within lick cycles.

In summary, these results show that DP and SP neurons in GC have higher stimulus responsiveness and licking rhythmicity relative to DI and SI. In addition, DP and SP neurons display greater spike-phase consistency compared with DI neurons.

\section{Taste-specific activity}

To assess the chemosensitivity of individual GC neurons, lickwarped PSTHs were used to decode taste identity (see Materials 
A

$$
\begin{aligned}
& \text { Representative Chemosensitive Neuron \#1 } \\
& \text { Narrow Tuning }
\end{aligned}
$$

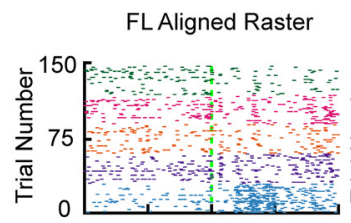

FL Aligned PSTH
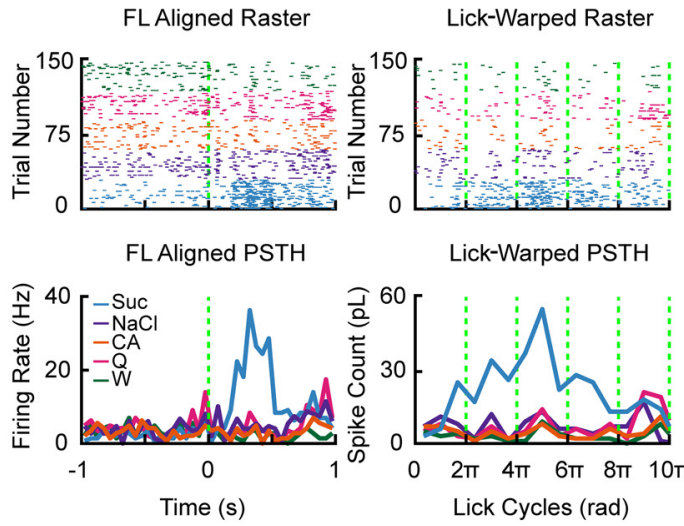

Lick-Warped PSTH

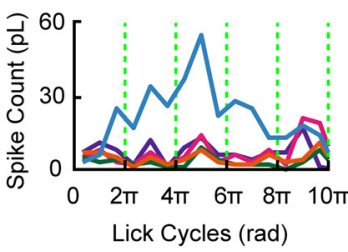

B

\section{Representative Chemosensitive Neuron \#2 Broad Tuning}

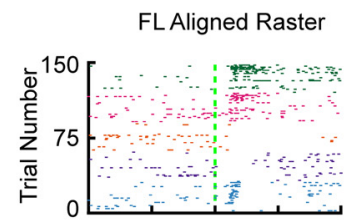

FL Aligned PSTH
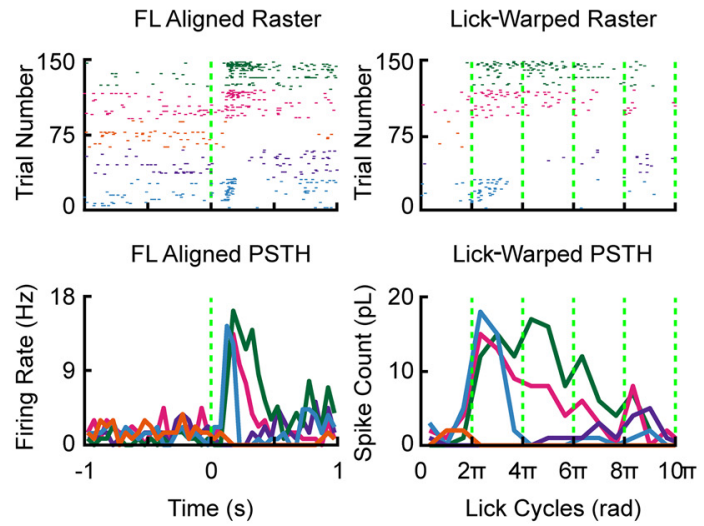
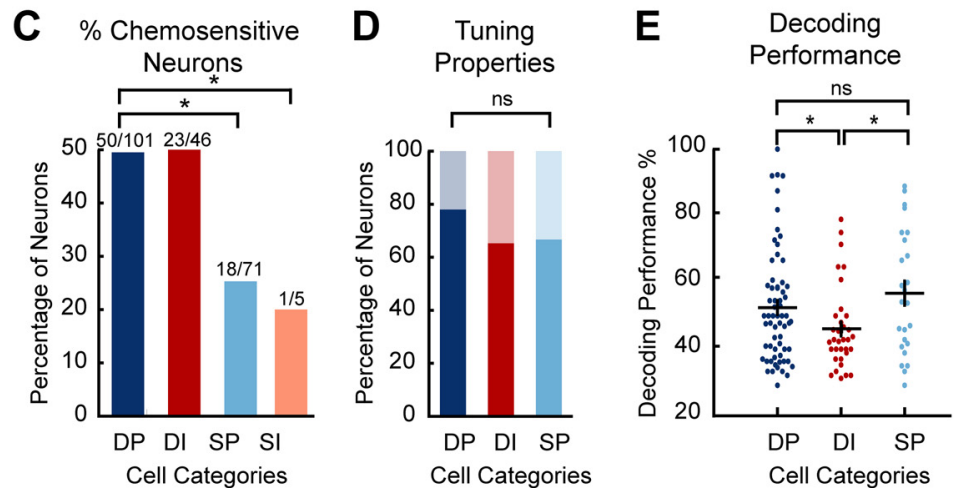

$\mathbf{F}$

Taste Coding Over Time

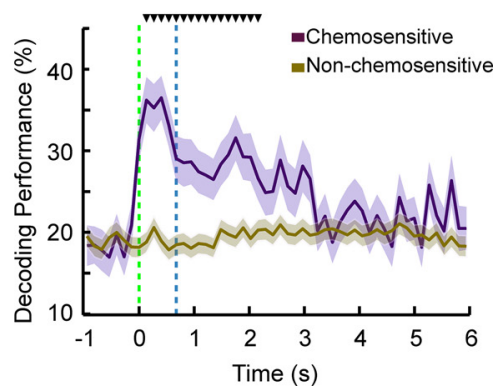

Figure 8. Taste coding in GC. A, Representative narrowly tuned chemosensitive GC neuron (DP) coding for sucrose. Left panel, First lick aligned raster (top) and PSTH (bottom) color coded for each tastant as shown in the inset. Right panel, Lick cycle warped activity of the same neuron with the raster (top) and PSTH (bottom) for the first five lick cycles following taste delivery. $\boldsymbol{B}$, Representative broadly tuned GC neuron (DP) coding for three tastants. Panels organized same as in $\boldsymbol{A}$. C, Percentage of chemosensitive neurons in each cell category. Asterisk denotes $p<0.05, \chi^{2}$ test. $\boldsymbol{D}$, Breadth of tuning across cell categories. Chemosensitive neurons classified as either narrow (1 taste decoded) or broadly tuned ( $>1$ taste decoded). No significant differences in distribution of narrow (dark colored) versus broadly tuned (light colored) neurons across cell types ( $p>0.05, \chi^{2}$ test). $\boldsymbol{E}$, Decoding performance across cell categories. Each dot represents the decoding performance of a successfully decoded tastant from a given cell category. Distributions compared using Kolmogorov-Smirnov test, asterisk indicates $p<0.05$ and ns indicates $p>0.05$. $\boldsymbol{F}$, Time course of average decoding performance across chemosensitive and non-chemosensitive neurons. Green dashed line marks the first lick cycle, blue dashed line marks the delivery of rinse. Solid lines show the mean decoding performance over time with SEM shaded around. Black triangles mark time bins where decoding of chemosensitive neurons was significantly different compared with the null distribution obtained from non taste-coding neurons. Significance of decoding performance compared with the chance level was computed using two-sided, unbalanced Mann-Whitney $U$ test with Dunn-Sidak correction.

and Methods, Taste coding analysis). A neuron was classified as chemosensitive if at least one tastant could successfully be decoded from its activity in the stimulus-evoked epoch. Figure $8 A, B$ shows two representative chemosensitive neurons. This analysis revealed that the relative percentage of chemosensitive neurons varied across cell categories with deep layers (DP, DI) showing greater percentage of chemosensitive neurons compared with superficial layer pyramidal neurons (DP: 54\% 50/101, DI: $50 \%$ 23/46, SP: $25 \% 18 / 71$, SI: $20 \% 1 / 5, p<0.01 \chi^{2}$ for DP, DI, SP, $p<0.01 \chi^{2}$ for all categories; Fig. $8 C$ ). We classified each chemosensitive neuron as either narrowly tuned (one taste successfully decoded) or broadly tuned (more than one taste successfully decoded). We found that neurons within DP, DI, and SP categories could show both narrow and broad tuning and that single GC neurons' activity could decode up to three different taste qualities. Next, we investigated whether distribution of narrow versus broadly tuned neurons varied across DP, DI, SP neurons (SI excluded because of low $n$ ). There were no significant differences across cell categories (DP: 78\%, 39/50 narrow vs $22 \%, 11 / 50$ broad, DI: $65 \%, 15 / 23$ narrow vs $34 \%, 8 / 23$ broad, SP: $67 \%, 12 / 18$ narrow vs $33 \%, 6 / 18$ broad, excluded SI: 1 narrow; Fig. $8 D$ ). We next assessed whether decoding performance (percentage of correctly classified trials over all trials of a given tastant) varied across chemosensitive neurons belonging to distinct cell categories. We compared performance for all successfully decoded tastants across DP, DI, and SP neurons. Decoding performance of DP $(52.5 \pm 2.1 \%)$ and SP $(56.8 \pm 3.7 \%)$ neurons were significantly higher than DI $(46 \pm 2.1 \%)$ neurons $(p<0.05$ for both, Kolmogorov-Smirnov test), but not different from one another ( $p=0.5$, Kolmogorov-Smirnov test) as shown in Figure $8 E$. We next investigated how well each tastant could be decoded and whether there were cell type-specific differences in responses to sucrose, salt, citric acid, quinine, and water. While our first analysis showed significant differences in how frequently each tastant was represented among all successfully decoded tastants (sucrose: $28 \%$, 35/124, salt: 18.6\%, 23/124, CA: 25\%, 31/124, Q: $15 \%, 19 / 124$, W: $\left.13 \%, 16 / 124, p<0.05, \chi^{2}\right)$, there were no significant differences in representation of different tastants across distinct cell type populations $\left(p=0.4, \chi^{2}\right)$. Furthermore, we found no differences in decoding performance across tastants $(p=0.9$, Kruskal-Wallis test).

To assess the time course of taste-specific information, we performed a time-resolved decoding analysis (see Materials and Methods). We found that for taste-coding neurons, taste 
selectivity peaks on the second lick and starts subsiding after the delivery of water on the sixth lick (Fig. $8 F$ ). Decoding performance of taste-coding neurons within the first five lick cycles (prewater drop) is significantly higher compared with the same length of time interval immediately following the water drop $(p<0.001$, two-sided, unbalanced Mann-Whitney $U$ test). Average taste-coding performance remains above chance level $2.2 \mathrm{~s}$ after the first lick onset, despite water drop being delivered on the sixth lick (which occurs $810 \mathrm{~ms}$ after the first lick on average). We compared the time course of decoding across cell categories and neurons with different functional properties (lick rhythmic vs non-rhythmic, pre-lick-modulated vs non-pre-lick modulated) and across different tastants and found no significant differences ( $p=$ N.S. for all comparisons, two-sided unbalanced Mann-Whitney $U$ test with Dunn-Sidak correction).

In summary, these results show that taste information peaks within $270 \mathrm{~ms}$ and persists for $2.2 \mathrm{~s}$ after the animals contact the taste solution. The data reveal a greater proportion of chemosensitive neurons in deep layers of GC, with balanced distribution of narrow and broadly tuned neurons across the cell categories. Furthermore, we observed a higher decoding performance for pyramidal neurons of deep and superficial layers compared with DI neurons. Overall, our results show layer and cell type-specific differences in representation of taste information across GC neurons.

\section{Convergence of different functional features in GC neurons}

Our results show that FRs of GC neurons can be modulated in the prestimulus period and, in the stimulus period, both by licking and by taste. Are these distinct features represented by different neurons or can they converge on the same cell? Representative examples of neurons that demonstrate convergence of two features based on the previously described criteria are plotted in Figure 8A,B. At the population level, 85.7\% (191/223) of all recorded neurons displayed at least one type of modulation and $56.5 \%$ of those (108/191) showed two or three types of modulations (Fig. 9C). The number of distinct modulations as a function of cell categories is shown in Figure $9 D\left(p<0.01, \chi^{2}\right)$, with deep layer neurons, DP and DI displaying very similar patterns. This convergence may reflect synergy among processes, as suggested by the higher proportion of taste-coding neurons in the group of those modulated during the prestimulus period (57/85) compared with those in the group of neurons not modulated before licking (35/88, $p<0.001, \chi^{2}$ test). Overall, these results show that more than half of the neurons recruited during the task can represent more than one feature.

\section{Discussion}

Our findings reveal how putative pyramidal and inhibitory neurons in deep and superficial layers of the GC show distinct FR modulations during different epochs of taste consumption in licking mice. At baseline, DI neurons had higher FRs compared with pyramidal neurons of deep and superficial layers (DP and SP) as well as SI neurons (albeit the small number of this group limits its interpretability). A larger proportion of DI neurons showed robust FR modulations before licking onset compared with other cell categories. These anticipatory modulations were aligned to the initiation of licking rather than the spout movement. MM analysis revealed that only $60 \%$ of prestimulusmodulated neurons showed significant correlations with MMs. Furthermore, neurons with significant cross-correlations displayed heterogeneous temporal relationships with MMs suggesting that prestimulus activity could not simply be a result of MMs that occur before lick initiation. During licking, we observed widespread rhythmic firing modulations in all GC cell categories, however, the precision of spike times within the lick cycle was much greater in deep and superficial layer pyramidal neurons. Next, we analyzed taste-evoked activity, using a taste stimulation paradigm. Upon presentation of tastants, GC neurons could respond to one or multiple taste qualities with no significant differences in the breadth of tuning of chemosensitive neurons across categories. While there were no differences in breath of tuning of chemosensitive neurons across cell categories, the proportion of GC taste-coding neurons was higher in deep layers compared with superficial layers. Finally, the distinct modulations (i.e., pre-lick modulations, licking rhythmicity, and taste-coding) could co-occur in single neurons and the convergence of these modulations did not vary in a layer and cell typedependent manner.

\section{Differences in baseline and stimulus-evoked FRs across layers and cell types}

Baseline and stimulus-evoked FRs were significantly different across cell categories, with DI displaying highest FRs at both epochs. Interestingly, while DP, SP, and SI neurons showed similar FRs at baseline, their activity became more dissimilar in the stimulus epoch with DP neurons showing significantly higher FRs than SP neurons. These observed differences in FRs of GC neurons could result from differences in intrinsic properties and/or in upstream connectivity and synaptic inputs across categories.

Layer-dependent differences in FRs are consistent with findings from other primary sensory cortices where neurons in superficial layers were found to fire much more sparsely than deep layer neurons (Niell and Stryker, 2008; Sakata and Harris, 2009; O'Connor et al., 2010). Both in vitro and in vivo studies have shown that superficial layer neurons are under greater inhibitory tone compared with deeper layers (Adesnik and Scanziani, 2010; Mateo et al., 2011; Adesnik et al., 2012; Beltramo et al., 2013; Haider et al., 2013; Petersen and Crochet, 2013), suggesting that the lower FRs in superficial layers could arise from this strong inhibitory tone. In fact, application of $\mathrm{GABA}_{\mathrm{A}}$ antagonist have been shown to increase responses in GC neurons (Ogawa et al., 1998). Cell type-dependent differences in FRs are also consistent with studies in other sensory cortices where inhibitory neurons displayed higher FRs compared with putative excitatory neurons (Niell and Stryker, 2008; Gentet et al., 2010). It is important to note that not all inhibitory neurons display high FRs and narrow waveforms and that spontaneous and evoked FRs can show distinctions across inhibitory neuron subtypes (Rudy et al., 2011).

It is worth noting that we have recorded from very few putative inhibitory neurons in the superficial layers compared with the deep layers. The first possible explanation is that there are fewer inhibitory neurons in the superficial layers of GC. The second possibility is that there are distinct subtypes of inhibitory neurons across GC laminae and the inhibitory neuron subtype (s) that are present in the superficial layers of GC cannot be classified readily with our method. In situ hybridization data (Lein et al., 2007) illustrate that inhibitory neurons indeed show laminarspecific distribution patterns in GC with vasoactive intestinal polypeptide (VIP)-expressing neurons predominantly occupying superficial layers and parvalbumin (PV)-expressing neurons showing denser distribution in the deeper layers. This layer-specific distribution has also been observed in primary 
A

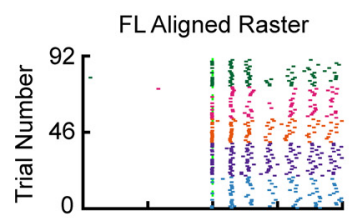

FL Aligned PSTH

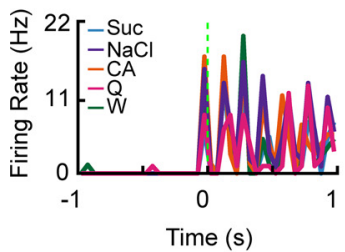

C

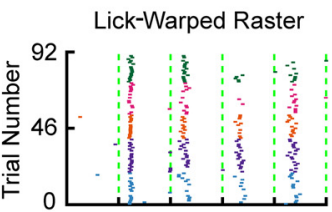

Lick-Warped PSTH

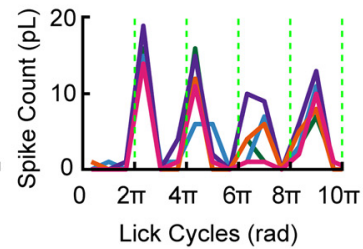

Convergence of Modulation Types

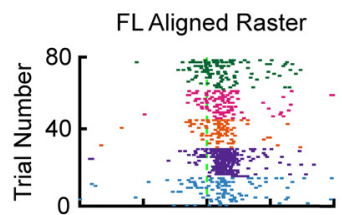

FL Aligned PSTH

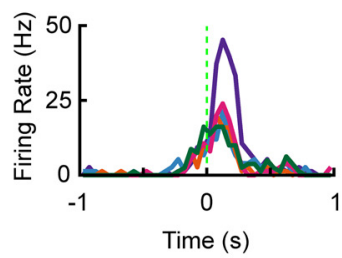

D

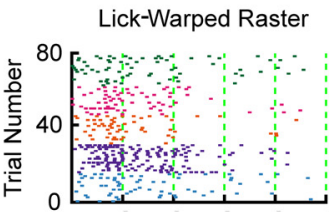

Lick-Warped PSTH

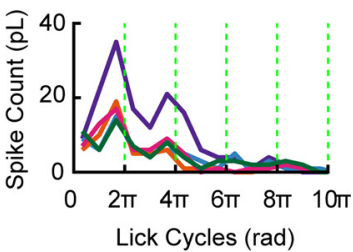

Cell Category-Specific Convergence

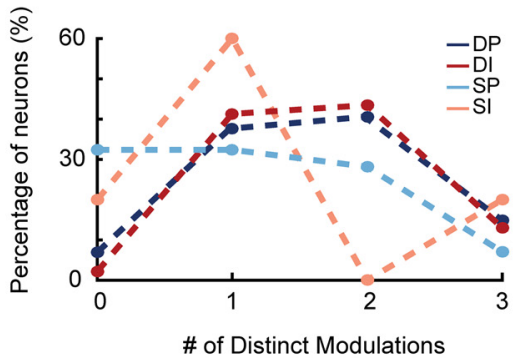

Figure 9. Convergence of distinct modulations on single $\mathrm{GC}$ neurons. Two representative neurons displaying convergence of distinct modulations are plotted in panels $\boldsymbol{A}, \boldsymbol{B}$. For $\boldsymbol{A}, \boldsymbol{B}$, left panels show the raster plot (top) and PSTH (bottom) of first lick aligned activity for all trials. Time 0 indicates first lick and is marked by green dashed line. Responses to five different taste trials are shown by distinct colors. Right panels, lick-warped raster (top) and PSTH (bottom) for the first five lick cycles occurring before the rinse; 0 radians on the $x$-axis indicates the onset of the first lick and consecutive lick cycles are marked as green dashed lines. A, Representative GC neuron (DP) that displays rhythmic licking and taste-specific modulation. $\boldsymbol{B}$, Representative GC neuron (DP) that displays excitatory prestimulus modulation, and taste-specific modulation. C, Venn diagram displaying the relative distribution of modulation types across all GC neurons that had at least one type of modulation (191/223). D, Distribution of neurons displaying zero (no modulation of any type), one, two, or three modulations for each cell category. The distributions are significantly different across the cell categories $\left(p<0.0001, \chi^{2}\right.$ test).

somatosensory cortex where PV neurons are concentrated in L4L6 and the 5HT3a neurons (which include the VIP neurons) are concentrated in L1-L3. It is possible that the lower FR of VIP neurons (Mesik et al., 2015) might lead to a sampling bias where they are less likely to be detected and sorted in extracellular recording configurations. The basis for low inhibitory neuron sampling in the superficial layers of GC would need to be further investigated using immunohistochemistry and slice electrophysiology techniques.

\section{Prestimulus modulations in GC}

We observed that $60 \%$ of all GC neurons display FR modulations beginning around $200 \mathrm{~ms}$ before animals' contact with taste solutions. This result is consistent with previous work in licking rodents (Yamamoto et al., 1988; Stapleton et al., 2006) and extends it by demonstrating that the prevalence of this phenomenon changes in a cell-specific manner. These modulations were most prevalent in DI neurons where they were largely excitatory. The small percentage of prestimulus-inhibited DI neurons displayed lower baseline FRs than prestimulus-excited DI neurons. It is possible that these DI neurons with distinct baseline FRs and prestimulus modulation patterns correspond to different subtypes of inhibitory neurons. Cell type-specific electrophysiology experiments from mouse sensory cortices shows that PV neurons display higher FRs compared with VIP neurons (Mesik et al.,
2015). Based on these findings it is possible to hypothesize that the larger proportion of DI neurons that show high baseline FRs and prestimulus excitation correspond to PV neurons whereas low baseline FR neurons that show prestimulus inhibition may correspond to VIP neurons. Studies monitoring the activity of a large number of distinct interneuron types will be needed to confirm this point.

Prestimulus modulations are not associated with spout movement, but are instead tightly aligned to timing of the first lick. To assess whether potential MMs could be the source of this, we investigated the cross-correlations between MMs and spiking activity. This analysis revealed that more than one third of prestimulus-modulated neurons did not display significant correlations with MMs. Furthermore, the subpopulation of neurons that did have significant correlations with MMs displayed complex temporal relationships. In the majority of cases, spike modulations preceded MMs. The complexity of the temporal relationship between MMs and GC spiking suggests that while the two can be correlated in a subset of neurons, it is unlikely that simple presence of MMs drives the observed prestimulus modulations in GC. Instead, pre-lick modulations could reflect anticipatory or premotor intentional signal or neuromodulatory/ attentional activity associated with the onset of a consummatory act.

What could be the functional role of these pre-lick modulations? The disproportionate recruitment of DI neurons before 
taste consumption suggests that this increased inhibitory tone could play a role in shaping subsequent taste responses. In fact, it has been shown in GC of anesthetized rodents that application of $\mathrm{GABA}_{\mathrm{A}}$ antagonist bicuculline can alter taste responses in a taste-specific manner (Ogawa et al., 1998). The role of inhibition in shaping sensory responses has been demonstrated in other primary sensory cortices (Wang et al., 2000; Wehr and Zador, 2003; Wilent and Contreras, 2005; Liu et al., 2010; Atallah et al., 2012; Lee et al., 2012; Wilson et al., 2012). It is worth noting that the pre-lick modulations demonstrated in our study are distinct from previously reported cue-evoked anticipatory responses in GC (Samuelsen et al., 2012, 2013; Gardner and Fontanini, 2014; Vincis and Fontanini, 2016). While cue-evoked responses are aligned to the onset of conditioned stimulus (i.e., auditory, visual or olfactory cue occurring as early as $2.5 \mathrm{~s}$ before taste delivery), pre-lick modulations occur right before licking initiation and are temporally segregated ( $1 \mathrm{~s})$ from the auditory tone in our task.

\section{FR modulations during licking}

The great majority of GC neurons (88\%) showed significant FR changes within the $500 \mathrm{~ms}$ interval following the first lick. These responses were predominantly excitatory in all categories with the relative prevalence of excitatory versus inhibitory modulations differing across cell categories. DI neurons showed the highest proportion of excitatory modulations, whereas SP neurons showed the highest proportion of inhibitory modulations in the stimulus-evoked epoch. Interestingly, SP neurons had the highest prevalence of inhibitory modulations in the prestimulus epoch as well. The inhibitory modulations observed in SP neurons in both prestimulus and stimulus-evoked epochs suggests a strong inhibitory control on SP neurons. These results are complimentary with our findings that SP neurons also show lower FRs than DP neurons in stimulus-evoked epoch.

Our results revealed that $44 \%$ of all GC neurons display rhythmic changes in FRs that correspond to the licking rhythm $(7 \mathrm{~Hz})$ during taste consumption. While such rhythmic activity was evident from PSTHs aligned to the first lick, lick-warped PSTHs revealed that in rhythmically-modulated DP and SP neurons, spikes occurred preferentially in specific phases of the lick cycles across trials. In contrast to DP and SP neurons, spikes of DI neurons, while also showing rhythmic modulations, were not occurring exclusively in a given phase of the lick cycle.

Licking is a rhythmic motor act that shapes timing of gustatory and somatosensory information arriving to the tongue. Given this robust influence of licking in patterning the gustatory input, it is not surprising that the neural activity along the taste pathway shows rhythmic FR modulations (Yamamoto et al., 1988; Gutierrez et al., 2006, 2010; Stapleton et al., 2006; Roussin et al., 2012; Weiss et al., 2014). The results presented here extend the previous findings by demonstrating that the licking induced rhythmicity of neural activity in GC is stronger in pyramidal neurons compared with inhibitory neurons. It is most likely that this rhythmic activity in GC is a continuation of the rhythmicity observed throughout the entire gustatory axis in licking animals (Stapleton et al., 2006; Gutierrez et al., 2010; Roussin et al., 2012). Given how consistent the spike phase is across lick cycles and trials, this effect is likely driven by a region that provides strong and extensive input to GC during taste consumption such as VPMpc. It is possible that the observed differences in spikephase fidelity across cell categories reflect a differential strength of this rhythmic input onto neurons within GC. Neurons that either do not receive direct innervation from the source of rhythmic activity, or receive sparse innervation are more likely to display weak rhythmicity and high trial to trial variability in spike phase.

When we analyzed taste responses across layers and cell types, we found a higher proportion of chemosensitive neurons in deep layers (both DP and DI) compared with superficial layers. The majority of chemosensitive neurons across all categories were narrowly tuned, but we did also observe a significant contingent of cells responding to multiple tastants. The presence of both narrowly and broadly tuned neurons in GC is consistent with previous findings in rats (Yamamoto et al., 1984; Ogawa et al., 1992; Katz et al., 2001; Stapleton et al., 2006; Jezzini et al., 2013) and more recently in mice (Fletcher et al., 2017; Bouaichi and Vincis, 2020). However, our results show higher prevalence of narrowly tuned neurons compared with some of the previous studies in alert rodents. This is likely the result of the design of our paradigm, in which mice receive a single drop of tastant at their first lick and a water rinse four dry licks later. This design, which has the advantage of standardizing licking across stimuli, relies on a limited amount of tastant followed by dry licks and a rinse, and shortens the temporal window available for tastecoding analysis. While the water rinse does not instantaneously extinguish taste coding (which continues to be significant up to $2.2 \mathrm{~s}$ following the first lick), it nevertheless interferes with the normal processing of taste. Amount of stimulus delivered and latency (and amount) of the rinse, are likely to have a significant impact on the analysis of taste responses. In fact, it has been shown that the number of fluid drops delivered can alter the responsiveness of NTS neurons in the gustatory pathway (Roussin et al., 2012) and that taste responses with rich temporal dynamics can emerge at latencies longer than those examined in this study (Katz et al., 2001; Roussin et al., 2012; Jezzini et al., 2013; Weiss et al., 2014; Bouaichi and Vincis, 2020).

A well-established temporal coding model of GC features a sequence of three coding epochs (i.e., somatosensory, chemosensory, and palatability) evolving over $2.5 \mathrm{~s}$ after stimulus. Our data are compatible with this model showing the presence of lickingrelated activity consistent with somatosensory signals, and chemosensory-related activity peaking at the second lick. While the structure of our paradigm did not allow us to explore the palatability epoch, recent results from (Bouaichi and Vincis, 2020) show its existence even in licking mice.

Interestingly, in our conditions, no difference in breadth of tuning was observed across layers. Contrary to the findings and theories from visual cortex where superficial neurons display increased stimulus selectivity compared with deep layers (Niell and Stryker, 2008), the results from GC show that superficial layers show a similar tuning curve compared with deep layers. While there were no differences across breadth of tuning, decoding analysis revealed that pyramidal neurons (both DP and SP) are better at encoding taste information compared with inhibitory neurons (DI). This difference across pyramidal and inhibitory neurons is consistent with findings from visual and somatosensory cortices where pyramidal neurons display stimulus-specific responses and inhibitory neurons display less specific activation (Cardin et al., 2007; Sohya et al., 2007; Kerlin et al., 2010).

\section{Convergence of response types in GC}

Our experiments demonstrate that neurons in GC can encode for different variables associated with tasting. They can show pre-lick modulations, licking-related rhythmicity and taste coding. Do neurons in GC act as integrators of these feature or as labeled-line processors of distinct dimensions? To address this 
question, we investigated to what extent single GC neurons could represent multiple features. The results revealed evidence for both specialized neurons encoding only one feature and integrators encoding two or more features. In general, the presence of specialized neurons suggests that each phenomenon represents a stand-alone signal, potentially arriving from distinct sources and can be processed independently, and not the manifestation of common signals. The majority of neurons with only one response type were modulated during the prestimulus period, emphasizing the relevance of this process for GC and its distinctiveness from licking-related activity. A large proportion of both prestimulus and lick rhythm modulated neurons could also code for taste information. In fact, pre-lick-modulated neurons were more likely to be taste coding than neurons not modulated in the pre-lick epoch, a result that suggests synergistic effects between the different processes. Integrating anticipatory, sensorimotor and gustatory information may have an obvious benefit for sensory coding, higher order function and learning in response to multimodal gustatory experiences. Analysis of the distribution of the various types of modulations did not show evidence of laminarity or preference for cell type, suggesting an even distribution of properties in GC. However, more studies will be required to ultimately address this issue. Altogether, our data emphasize how GC uses a mixed strategy to process multiple dimensions associated with tasting, combining feature selectivity with mixed coding and integrating anticipatory activation, licking, and taste coding.

\section{References}

Adesnik H, Scanziani M (2010) Lateral competition for cortical space by layer-specific horizontal circuits. Nature 464:1155-1160.

Adesnik H, Bruns W, Taniguchi H, Huang ZJ, Scanziani M (2012) A neural circuit for spatial summation in visual cortex. Nature 490:226-231.

Atallah BV, Bruns W, Carandini M, Scanziani M (2012) Parvalbuminexpressing interneurons linearly transform cortical responses to visual stimuli. Neuron 73:159-170.

Beltramo R, D'Urso G, Dal Maschio M, Farisello P, Bovetti S, Clovis Y, Lassi G, Tucci V, De Pietri Tonelli D, Fellin T (2013) Layer-specific excitatory circuits differentially control recurrent network dynamics in the neocortex. Nat Neurosci 16:227-234.

Bouaichi CG, Vincis R (2020) Cortical processing of chemosensory and hedonic features of taste in active licking mice. J Neurophysiol 123:19952009.

Cardin JA, Palmer LA, Contreras D (2007) Stimulus feature selectivity in excitatory and inhibitory neurons in primary visual cortex. J Neurosci 27:10333-10344.

Chen X, Gabitto M, Peng Y, Ryba NJ, Zuker CS (2011) A gustotopic map of taste qualities in the mammalian brain. Science 333:1262-1266.

Cohen JY, Haesler S, Vong L, Lowell BB, Uchida N (2012) Neuron-type-specific signals for reward and punishment in the ventral tegmental area. Nature 482:85-88.

Crochet S, Poulet JF, Kremer Y, Petersen CC (2011) Synaptic mechanisms underlying sparse coding of active touch. Neuron 69:1160-1175.

de Kock CP, Bruno RM, Spors H, Sakmann B (2007) Layer- and cell-typespecific suprathreshold stimulus representation in rat primary somatosensory cortex. J Physiol 581:139-154.

Fletcher ML, Ogg MC, Lu L, Ogg RJ, Boughter JD Jr (2017) Overlapping representation of primary tastes in a defined region of the gustatory cortex. J Neurosci 37:7595-7605.

Gallistel CR, Fairhurst S, Balsam P (2004) The learning curve: implications of a quantitative analysis. Proc Natl Acad Sci USA 101:13124-13131.

Gardner MP, Fontanini A (2014) Encoding and tracking of outcome-specific expectancy in the gustatory cortex of alert rats. J Neurosci 34:1300013017.

Gentet LJ, Avermann M, Matyas F, Staiger JF, Petersen CCH (2010) Membrane potential dynamics of GABAergic neurons in the barrel cortex of behaving mice. Neuron 65:422-435.
Gilbert CD (1977) Laminar differences in receptive field properties of cells in cat primary visual cortex. J Physiol 268:391-421.

Graham DM, Sun C, Hill DL (2014) Temporal signatures of taste quality driven by active sensing. J Neurosci 34:7398-7411.

Gutierrez R, Carmena JM, Nicolelis MA, Simon SA (2006) Orbitofrontal ensemble activity monitors licking and distinguishes among natural rewards. J Neurophysiol 95:119-133.

Gutierrez R, Simon SA, Nicolelis MA (2010) Licking-induced synchrony in the taste-reward circuit improves cue discrimination during learning. J Neurosci 30:287-303.

Haider B, Hausser M, Carandini M (2013) Inhibition dominates sensory responses in awake cortex. Nature 493:97-100.

Jezzini A, Mazzucato L, Camera GL, Fontanini A (2013) Processing of hedonic and chemosensory features of taste in medial prefrontal and insular networks. J Neurosci 33:18966-18978.

Jones LM, Fontanini A, Sadacca BF, Miller P, Katz DB (2007) Natural stimuli evoke dynamic sequences of states in sensory cortical ensembles. Proc Natl Acad Sci USA 104:18772-18777.

Katz DB, Simon SA, Nicolelis MA (2001) Dynamic and multimodal responses of gustatory cortical neurons in awake rats. J Neurosci 21: 4478-4489.

Kerlin AM, Andermann ML, Berezovskii VK, Reid RC (2010) Broadly tuned response properties of diverse inhibitory neuron subtypes in mouse visual cortex. Neuron 67:858-871.

Krupa DJ, Wiest MC, Shuler MG, Laubach M, Nicolelis MA (2004) Layerspecific somatosensory cortical activation during active tactile discrimination. Science 304:1989-1992.

Lee SH, Kwan AC, Zhang S, Phoumthipphavong V, Flannery JG, Masmanidis SC, Taniguchi H, Huang ZJ, Zhang F, Boyden ES, Deisseroth K, Dan Y (2012) Activation of specific interneurons improves V1 feature selectivity and visual perception. Nature 488:379-383.

Lein ES, Hawrylycz MJ, Ao N, Ayres M, Bensinger A, Bernard A, Boe AF, Boguski MS, Brockway KS, Byrnes EJ, Chen L, Chen L, Chen TM, Chin MC, Chong J, Crook BE, Czaplinska A, Dang CN, Datta S, Dee NR, et al. (2007) Genome-wide atlas of gene expression in the adult mouse brain. Nature 445:168-176.

Liu BH, Li P, Sun YJ, Li YT, Zhang LI, Tao HW (2010) Intervening inhibition underlies simple-cell receptive field structure in visual cortex. Nat Neurosci 13:89-96.

Liu H, Fontanini A (2015) State dependency of chemosensory coding in the gustatory thalamus (VPMpc) of alert rats. J Neurosci 35:15479-15491.

Martinez LM, Alonso JM, Reid RC, Hirsch JA (2002) Laminar processing of stimulus orientation in cat visual cortex. J Physiol 540:321-333.

Mateo C, Avermann M, Gentet LJ, Zhang F, Deisseroth K, Petersen CC (2011) In vivo optogenetic stimulation of neocortical excitatory neurons drives brain-state-dependent inhibition. Curr Biol 21:1593-1602.

Mesik L, Ma WP, Li LY, Ibrahim LA, Huang ZJ, Zhang LI, Tao HW (2015) Functional response properties of VIP-expressing inhibitory neurons in mouse visual and auditory cortex. Front Neural Circuits 9:22.

Niell CM, Stryker MP (2008) Highly selective receptive fields in mouse visual cortex. J Neurosci 28:7520-7536.

O'Connor DH, Peron SP, Huber D, Svoboda K (2010) Neural activity in barrel cortex underlying vibrissa-based object localization in mice. Neuron 67:1048-1061.

Ogawa H, Hasegawa K, Murayama N (1992) Difference in taste quality coding between two cortical taste areas, granular and dysgranular insular areas, in rats. Exp Brain Res 91:415-424.

Ogawa H, Hasegawa K, Otawa S, Ikeda I (1998) GABAergic inhibition and modifications of taste responses in the cortical taste area in rats. Neurosci Res 32:85-95.

Petersen CC, Crochet S (2013) Synaptic computation and sensory processing in neocortical layer 2/3. Neuron 78:28-48.

Poo C, Isaacson JS (2009) Odor representations in olfactory cortex: "sparse" coding, global inhibition, and oscillations. Neuron 62:850-861.

Reyes-Puerta V, Sun JJ, Kim S, Kilb W, Luhmann HJ (2015) Laminar and columnar structure of sensory-evoked multineuronal spike sequences in adult rat barrel cortex in vivo. Cereb Cortex 25:2001-2021.

Roussin AT, D'Agostino AE, Fooden AM, Victor JD, Di Lorenzo PM (2012) Taste coding in the nucleus of the solitary tract of the awake, freely licking rat. J Neurosci 32:10494-10506. 
Rudy B, Fishell G, Lee S, Hjerling-Leffler J (2011) Three groups of interneurons account for nearly $100 \%$ of neocortical GABAergic neurons. Dev Neurobiol 71:45-61.

Sakata S, Harris KD (2009) Laminar structure of spontaneous and sensoryevoked population activity in auditory cortex. Neuron 64:404-418.

Samuelsen CL, Gardner MP, Fontanini A (2012) Effects of cue-triggered expectation on cortical processing of taste. Neuron 74:410-422.

Samuelsen CL, Gardner MP, Fontanini A (2013) Thalamic contribution to cortical processing of taste and expectation. J Neurosci 33:1815-1827.

Sohya K, Kameyama K, Yanagawa Y, Obata K, Tsumoto T (2007) GABAergic neurons are less selective to stimulus orientation than excitatory neurons in layer II/III of visual cortex, as revealed by in vivo functional Ca2 + imaging in transgenic mice. J Neurosci 27:2145-2149.

Stapleton JR, Lavine ML, Wolpert RL, Nicolelis MA, Simon SA (2006) Rapid taste responses in the gustatory cortex during licking. J Neurosci 26:4126-4138.

Stapleton JR, Lavine ML, Nicolelis MA, Simon SA (2007) Ensembles of gustatory cortical neurons anticipate and discriminate between tastants in a single lick. Front Neurosci 1:161-174.

Vincis R, Fontanini A (2016) Associative learning changes cross-modal representations in the gustatory cortex. Elife 5.
Wang J, Caspary D, Salvi RJ (2000) GABA-A antagonist causes dramatic expansion of tuning in primary auditory cortex. Neuroreport 11:1137-1140.

Wehr M, Zador AM (2003) Balanced inhibition underlies tuning and sharpens spike timing in auditory cortex. Nature 426:442-446.

Weiss MS, Victor JD, Di Lorenzo PM (2014) Taste coding in the parabrachial nucleus of the pons in awake, freely licking rats and comparison with the nucleus of the solitary tract. J Neurophysiol 111:1655-1670.

Wilent WB, Contreras D (2005) Dynamics of excitation and inhibition underlying stimulus selectivity in rat somatosensory cortex. Nat Neurosci 8:1364-1370.

Wilson NR, Runyan CA, Wang FL, Sur M (2012) Division and subtraction by distinct cortical inhibitory networks in vivo. Nature 488:343-348.

Yamamoto T, Yuyama N, Kato T, Kawamura Y (1984) Gustatory responses of cortical neurons in rats. I. Response characteristics. J Neurophysiol 51:616-635.

Yamamoto T, Matsuo R, Kiyomitsu Y, Kitamura R (1988) Sensory inputs from the oral-region to the cerebral-cortex in behaving rats - an analysis of unit responses in cortical somatosensory and taste areas during ingestive behavior. J Neurophysiol 60:1303-1321.

Yokota TK, Eguchi K, Hiraba K (2011) Functional properties of putative pyramidal neurons and inhibitory interneurons in the rat gustatory cortex. Cereb Cortex 21:597-606. 